To appear in Neural Computation, accepted January 31, 2002.

\title{
Independent Components of Magnetoencephalography: Localization
}

\author{
Akaysha C. Tang, ${ }^{123 *}$ Barak A. Pearlmutter, ${ }^{23}$ \\ Natalie A. Malaszenko, Dan B. Phung, Bethany C. Reeb \\ Departments of ${ }^{1}$ Psychology, ${ }^{2}$ Computer Science, ${ }^{3}$ Neurosciences \\ University of New Mexico, Albuquerque, NM 87131, USA
}

January 31,2002

\begin{abstract}
We applied second-order blind identification (SOBI), an independent component analysis (ICA) method, to MEG data collected during cognitive tasks. We explored SOBI's ability to help isolate underlying neuronal sources with relatively poor signal-to-noise ratios, allowing their identification and localization. We compare localization of the SOBI-separated components to localization from unprocessed sensor signals, using an equivalent current dipole (ECD) modeling method. For visual and somatosensory modalities, SOBI preprocessing resulted in components that can be localized to physiologically and anatomically meaningful locations. Furthermore, this preprocessing allowed the detection of neuronal source activations that were otherwise undetectable. This increased probability of neuronal source detection and localization can be particularly beneficial for MEG studies of higher level cognitive functions, which often have greater signal variability and degraded signal-to-noise ratios than sensory activation tasks.
\end{abstract}

\section{Introduction}

Magnetoencephalography (MEG) is a passive functional brain imaging technique which, under ideal conditions, can monitor the activation of a neuronal population with a spatial resolution of a few mm and with millisecond temporal resolution (Hamalainen et al., 1993; George et al., 1995). Typical signals associated with neuronal activity are on the order of one hundred fT, while the noise signals within a shielded room tend to be much larger (Lewine and Orrison, 1995). Furthermore, the intrinsic sensor noise is comparable in magnitude to small neuronal signals. Therefore, what the sensors record during an experiment is always a mixture of small neuromagnetic and large

*Corresponding author: Dept of Psychology, Logan Hall, University of New Mexico, Albuquerque, NM 87131, 505 277-4025 (voice), 505 277-1394 (fax), akaysha@unm.edu. 
noise signals. This relatively poor signal-to-noise ratio ${ }^{1}$ can affect the localization of neuronal activity.

Several independent component analysis (ICA) algorithms, such as second-order blind identification (SOBI) (Belouchrani et al., 1993; Cardoso, 1994), Bell and Sejnowski (1995) Infomax, and fICA (Hyvärinen and Oja, 1997), have been applied to EEG data (Makeig et al., 1996, 1997, 1999b; Jung et al., 2000a,b) and MEG data (Vigário et al., 1998; Tang et al., 2000a; Vigário et al., 1999, 2000; Wübbeler et al., 2000; Ziehe et al., 2000; Cao et al., 2000). In both applications, ICA methods have proven useful for artifact removal and for improving the signal-to-noise ratio (Jung et al., 2000a,b; Vigário et al., 1998; Tang et al., 2000a). For general reviews of ICA see Amari and Cichocki (1998); Cardoso (1998); Hyvärinen (1999); Vigário et al. (2000).

For MEG, in addition to separating various noise signals from the neuromagnetic signals, SOBI and fICA have been shown to separate one neuronal source from another between and within the same modality (Tang et al., 2000a; Vigário et al., 1999, 2000). To localize functionally independent neuronal sources or to simultaneously localize and recover the time course of these neuronal sources, a variety of algorithms have been proposed (Mosher et al., 1992; Kinouchi et al., 1996; Sekihara et al., 1997; Nagano et al., 1998; Mosher and Leahy, 1998; Uutela et al., 1998; Mosher and Leahy, 1999; Schwartz et al., 1999; Sekihara et al., 2000; Huang et al., 2000; Aine et al., 2000; Ermer et al., 2000; Cao et al., 2000; Schmidt et al., 1999). Given their capability to separate noise and neuronal signals, ICA algorithms are expected to benefit all source localization methods by providing them with input signals that are more likely to be associated with functionally independent neuronal sources.

It was found, however, that the fICA-separated components yielded localization results qualitatively similar to those arrived at without ICA preprocessing (Vigário et al., 1999). Consequently, no substantial benefits from ICA were reported for neuromagnetic source localization. As one of the strengths of ICA is its ability to separate noise from the signals of interests, whether ICA could offer any advantage in source localization should depend on the signal-to-noise ratio in the sensor data. The experiment reported by Vigário et al. (1999) was optimally designed to produce strong and focal activation of a small number of neuromagnetic sources, and therefore high signal-tonoise ratios. Under such optimal conditions, ICA could not improve much upon the already good localization provided by conventional methods.

In this paper, we applied ICA to neuromagnetic signals with relatively poor signal-to-noise ratios collected during cognitive tasks involving large trial-to-trial variability in neuronal source activation and from a much larger number of sources. We localized these neuronal sources using the equivalent current dipole (ECD) modeling method (Neuromag) on SOBI-separated components, and on unprocessed sensor data. We found that SOBI preprocessing resulted in the localization of neuronal sources that could not be found when the dipole fitting method was directly applied to the sensor data. In addition, the process of localizing separated components required significantly less subjective judgment regarding which sensors to exclude from the analysis ${ }^{2}$ and at what time the dipoles are fitted. We suggest that ICA methods can be particularly effective and efficient in the study of higher level cognitive functions when the neuronal source activations are

\footnotetext{
${ }^{1}$ Unless otherwise indicated, we use signal-to-noise ratio in the sense defined in signal detection theory. Signals refer to the neuromagnetic signal of interest. Noise refers to all other signals including environmental and sensor noise and other background brain signals.

${ }^{2}$ It is a common practice to select $20-30$ sensors over the brain region of interest for dipole fitting.
} 
often characterized by their greater degree of variability and lower signal-to-noise ratios.

\section{Methods}

\subsection{Cognitive Tasks}

We collected MEG data from four right-handed subjects (two females and two males) during four visual reaction time tasks originally designed to study temporal lobe memory functions (Tang et al., 2000b). These tasks are described in detail in Appendix A. Here, we offer a brief description. In each task, a pair of colored patterns, one of which was the target, was presented on the left and right halves of the display screen. The subject was instructed to press either the left or right button when the target appeared on the left or right, respectively. In all tasks, the target was not described to the subject prior to the experiment. The subject was to discover the target by trial and error using auditory feedback (low and high tones corresponded to correct and incorrect responses, respectively). All subjects were able to discover the rule within a few trials.

The tasks differed in the memory load required for determining which of the pair is the target. Task one served to familiarize the subjects with all visual patterns. The subjects simply viewed the stimuli and were asked to press either the left or right button at their own choice while making sure approximately equal numbers of left and right button presses were performed. As such, task one placed little memory demand on the subject. Task two involved remembering a single target pattern which appeared on each trial paired with another pattern. Subjects pressed the right or left button to indicate whether the target pattern was on the left or the right. Task three involved remembering multiple targets, each always paired with the same non-target. Task four was the most complex. In task four, targets were context sensitive in a circular fashion, as in the game rock-paper-scissors. The amount of cognitive processing beyond the initial sensory processing increased successively from task one to task four.

We used data from these complex cognitive tasks to evaluate the capability of SOBI (see Appendix B.1) because of the relatively poor signal-to-noise ratios involved in comparison to sensory activation tasks. Specifically, these tasks involved (1) large visual field stimulation without the use of fixation points, (2) incidental somatosensory stimulation as a result of button presses during reaction time tasks, and (3) highly variable button press responses because precisely what form of the thumb movement should be, how the mouse was held, and where the hands rest were not specified. These sources of variability in visual and somatosensory activation can lead to poor signal-to-noise ratios in the average responses, making it particularly difficult to localize the neuronal sources from unprocessed averaged sensor data. The involvement of higher level cognitive functions, memory demands, and the small number of trials (90 in most cases) collected under each task condition, further decreased the signal-to-noise ratios in the averaged sensor data. These tasks therefore offered a set of challenging datasets in which the advantages of ICA methods could be revealed.

\subsection{Selection of ICA Methods}

In selection of ICA algorithms, one important consideration is the robustness of the algorithm to sensor noise. Instantaneous and summary algorithms are two extremes of ICA algorithms that 
differ in whether each point in time is considered in isolation. Instantaneous algorithms, such as Bell-Sejnowski Infomax (1995) and fICA (Hyvärinen and Oja, 1997), make repeated passes through the dataset and update the unmixing matrix in response to the data at each time point. They are derived under the assumption that the signals are white, and their results should therefore be invariant to shuffling of the data. As a consequence of this, they cannot take advantage of the temporal structure of each source as a cue for correct separation. In contrast, summary algorithms first make a pass through the data while summary statistics are accumulated by averaging; they then operate solely upon the summary statistics to find the separation matrix. Some summary algorithms collect statistic that allow them to make use of the temporal structure of the sources as a cue for separation. More importantly, summary algorithms should in general be relatively insensitive to sensor noise, because their summary statistics are averages over time. The relatively poor signal-to-noise ratios in MEG data suggested the choice of a summary algorithm rather than an instantaneous algorithm.

When it can be assumed that each source has a broad autocorrelation function, as is the case with brain signals, the summary algorithm SOBI (Belouchrani et al., 1993; Cardoso, 1994) can use this temporal structure as a cue and give high quality separation while imposing rather modest computational requirements. SOBI extracts a large set of statistics from the dataset, which it uses for the separation. Each of these statistics is calculated by averaging across the dataset, which makes the algorithm robust against noise. The particular statistics calculated are the correlations between pairs of sensors at a fixed delay, $\left\langle x_{i}(t) x_{j}(t+\tau)\right\rangle$. This makes good use of abundant but noisy data, and most importantly, SOBI can be tuned by modifying its set of delays (see Appendix B), allowing its users to gently integrate a very weak form of prior knowledge, namely knowledge of the length constant of the autocorrelation function. Although Bell-Sejnowski Infomax and fICA have been previously applied to MEG and EEG data, and other ICA algorithms, such as Contextual ICA (Pearlmutter and Parra, 1996) and Sparse Decomposition (Zibulevsky and Pearlmutter, 2001), are locally available, we selected SOBI as our ICA method based on the above properties of SOBI. However, we have not conducted a systematic comparison of ICA methods for our MEG data.

\subsection{Second-Order Blind Identification}

SOBI is considered blind as it makes no assumptions about the form of the mixing process. In other words, SOBI does not attempt to solve the inverse problem or use the physics of the situation in any way. It does not try to estimate currents, or know about Maxwell's equation or any of its consequences. The only physical assumption made about the mixing process is that it is instantaneous and linear.

Let $\mathbf{x}(t)$ be an $n$-dimensional vector of sensor signals, which we assume to be an instantaneous linear mixture of $n$ unknown independent underlying sources $s_{i}(t)$, via the unknown stationary $n \times n$ mixing matrix $\mathbf{A}$,

$$
\mathbf{x}(t)=\mathbf{A} \mathbf{s}(t)
$$

The ICA problem is to recover $\mathbf{s}(t)$, given the measurements $\mathbf{x}(t)$ and nothing else. This is accomplished by finding a matrix $\mathbf{W}$ which approximates $\mathbf{A}^{-1}$, up to permutation and scaling of its rows. SOBI assumes that the sources are statistically independent in time, and not necessarily orthogonal 
in space. It finds $\mathbf{W}$ by minimizing the correlation ${ }^{3}$ between one recovered source at time $t$ and another at time $t+\tau$.

The particular set of delays $\tau$ we used were chosen to cover a reasonably wide interval without extending beyond the support of the autocorrelation function. Measured in units of samples, at our $300 \mathrm{~Hz}$ sampling rate, the delays ${ }^{4}$ were

$$
\begin{aligned}
\tau \in\{ & 1,2,3,4,5,6,7,8,9 \\
& 10,12,14,16,18 \\
& 20,25,30,35,40,45,50,55 \\
& 60,65,70,75,80,85,90,95,100\} .
\end{aligned}
$$

Each recovered $s_{i}(t)$ also has a sensor space projection that gives the sensor readings of $s_{i}(t)$ (see Sec. B.2). This sensor projection can be displayed as a field map, and can be used as input to source localization algorithms. For example, after calculating its sensor projection, we can repackage a component for localization by Neuromag dipole modeling tools.

SOBI shares a number of weaknesses with all ICA methods: they all assume that there are as many sensors as sources; they all make some sort of independence assumption; they all assume that the mixing process is linear; and they all assume that the mixing process is stable. See Section 4.3 for further discussion.

\subsection{Localization of Separated Components}

SOBI was performed on continuous ${ }^{5}$ 122-channel data collected during the entire period of the experiment, sampled at $300 \mathrm{~Hz}$, and band-pass filtered at $0.03-100 \mathrm{~Hz}$. It generated 122 components, ${ }^{6}$ each a one-dimensional time series with an associated field map (see Appendix B.2). Each component potentially corresponds to a set of magnetic field generators.

Event triggered averages were calculated from their continuous single-trial time series for all 122 separated components, where the triggering events were either sensory stimuli or behavioral responses. For the specific tasks used here, there were typically 10-20 components in each experiment which showed responses locked to either stimuli or to button presses. Those with stimulusor motor-locked responses were candidate neuronal generators, since they showed task related activation. Those with responses locked onto other external events, such as eye blinks or heart beats (detected using EOG and EKG), were considered known noise sources. The rest were treated as non-task-related noise sources.

For a task related component, if its field map and time course were consistent with known neurophysiological and neuroanatomical facts, we considered it a neuronal component reflecting the activity of a neuronal generator. For example, if the field map of a component shows activation

\footnotetext{
${ }^{3}$ For justification for this minimization, see Discussion.

${ }^{4}$ The choice of delays can affect the results of separation. Depending on the types of sources activated by the behavioral task, the selection of delays can have complex interactions with the latency of evoked responses. This is an important topic and deserves a separate study.

${ }^{5}$ Note that ICA algorithms can also be applied to cross-trial averages rather than continuous data, as in Makeig et al. (1999c).

${ }^{6}$ ICA algorithms produce the same number of components as there are channels in their input.
} 
over the occipital cortex and the visual stimulus triggered average for this component contains an evoked response that peaks between $50-100 \mathrm{~ms}$, then it is considered to reflect the activity of a visual source in the occipital lobe. Using this procedure, neuronal and non-neuronal generators were separated and identified (Tang et al., 2000a,b). A dipole fitting method was then applied to the identified neuronal components. The input to the dipole fitting algorithm (Neuromag, xfit, least square) was the field map, and the output was the location of ECDs projected onto the subject's structural MRI images.

This same dipole fitting algorithm was used for localization with and without SOBI preprocessing. Because our goal was to evaluate whether ICA methods can improve source localization, we were not concerned with whether the least square dipole fitting was as good as more recent more sophisticated source modeling methods. Our interest was not in localization accuracy per se, but in the comparative performance of a given localization method when used alone, as opposed to being coupled with ICA.

In statistical comparison, to match the common practice in source modeling without SOBI, a subset of channels (20-30) over the region of interest were selected for dipole fitting with both methods. To localize each separated component, we chose channels over the region of interest showing stronger responses to the source. For localization without SOBI (the conventional practice) we began with the channels selected for SOBI localization, and then modified the selections to obtain a more dipolar field pattern. ${ }^{7}$ If these modifications improved the results then we used them, otherwise we used the original channel selections. This procedure gave the conventional practice an advantage because the event-triggered-average responses were cleaner in the separated components than in the raw data. In fact, the raw data were often so noisy that no channels could have been selected by following the same procedure on the raw data, and therefore no localization could have been performed without the channel selection information enabled by SOBI.

To localize a component, we used its field map as input to the dipole fitting program. ${ }^{8}$ One can select any time during the average time window to fit the dipole because the dipole solution for a component is invariant to time (see Sec. B.2). This independence of localization results from the dipole fitting time can significantly simplify the dipole localization process, making it less subjective than dipole localization directly from the sensor data, without the use of ICA. Using the conventional method, the time at which a dipole was fitted affects the final estimated dipole location.

To localize neuronal sources without SOBI preprocessing, we used event triggered sensor data (averages) as inputs to the dipole fitting program. We first chose the time with the largest evoked response amplitude within the time window of interest. Then a subset of channels (20-30) over the region of interest were selected. When the contour maps were single-dipolar for the selected channels at the time chosen, a single dipole fit was performed. Otherwise multiple dipoles were fitted. For details of the process, see the xfit manual. In the examples shown in the figures, all channels were used in the dipole fitting to show that SOBI can identify dipolar sources without any channel selection.

\footnotetext{
${ }^{7}$ A field map is judged dipolar by visual inspection (Hari and Salmelin, 1997). If it contains two sets of concentric contour lines, the field is considered dipolar. If it contains more or less than two sets, the field is not dipolar.

${ }^{8}$ Theoretically, one sampling point in time across all sensors contains all information about a source. In practice the Neuromag software needs a time series of at least several samples. Therefore, we calculated the event-triggered average for the component of interest and made an input .fif file containing the average.
} 


\section{Results}

\subsection{SOBI decomposition: time courses and sensor projections}

Using SOBI, continuous MEG signals from 122 channels were separated into 122 components. Each of these components has a time course and an associated sensor projection. The time course can be averaged across multiple trials using either the visual stimulus onset or the button press as a trigger. It can also be displayed as an $M E G$ image (e.g. Fig. 6, right), a pseudo-colored bitmap in which the responses of a given component during an entire experiment can be parsimoniously displayed (Jung et al., 1999). Typically, each row represents one discrete trial of stimulation and multiple trials are ordered vertically from top to bottom. See Sec. B.3 for details on the process of giving sensible units to the components. As shown in the overlay plots of the visual stimulus and button press triggered averages for all 122 components (Fig. 1cd), only a small fraction of the components showed task related responses. For clarity, these task related components are shown separately in Fig. 1a,b.

The components can be displayed in the sensor domain in field maps Fig. 6, left) or in a fullview graph using the Neuromag software xfit (Fig. 2-5). The sensor projections for two components are shown: one for a visual component (Fig. 2) and the other for a sensory-motor component (Fig. 3). It is clear that the two components are projected selectively to sensors over the visual and sensorymotor cortices. For comparison, the fullview plots of the sensor projection from the raw data (mixture of all components) are shown in Fig. 4-5.

\subsection{Energy in Separated Components}

We divided components into the following six categories: visual, somatosensory, ocular artifacts, $60 \mathrm{~Hz}$, sensor jumps, and other. ${ }^{9}$ Visual and somatosensory components were identified by their clearly visible evoked responses in the MEG images and by their activation patterns in the field maps (see following sections). These neuronal components were further verified by the consistency between the response latency, shown in the MEG images, and the spatial location of sensor activation, shown in the field maps (see subsequent sections). Ocular artifact sources were identified by their characteristic activation patterns in the field map and their large amplitude responses in the MEG image (Fig. 6a), which match signals measured by EOG (not shown). The $60 \mathrm{~Hz}$ components were identified by the clearly visible $60 \mathrm{~Hz}$ cyclic activity in the MEG images in Fig. 6b (see also Tang et al. (2000a)). Sensor jumps components were easily identified by the single-sensor activation in the field maps and sometimes by high contrast lines or dots in the MEG images (Fig. 6c).

For these five types of identified components, we calculated the amount of energy in each (see Appendix B.4), across all subjects and all tasks, using a window of $200 \mathrm{~ms}$ after either the visual stimulus presentation or the button presses ${ }^{10}$ (see Sec. B.4). This window was chosen to cover all neuronal responses. The amount of energy in a single component varied widely, between $0.17 \%$ and $71 \%$ of the total energy across all sensors. This range differed among the five categories of

\footnotetext{
${ }^{9}$ Sensor jumps refer to a peculiar property of the SQUID sensors which cause an enormous and nearly instantaneous DC shift. "Other" includes any components that do not belong to the first five categories.

${ }^{10}$ Note: window specification will affect the calculated energy.
} 
g replacements

g replacements
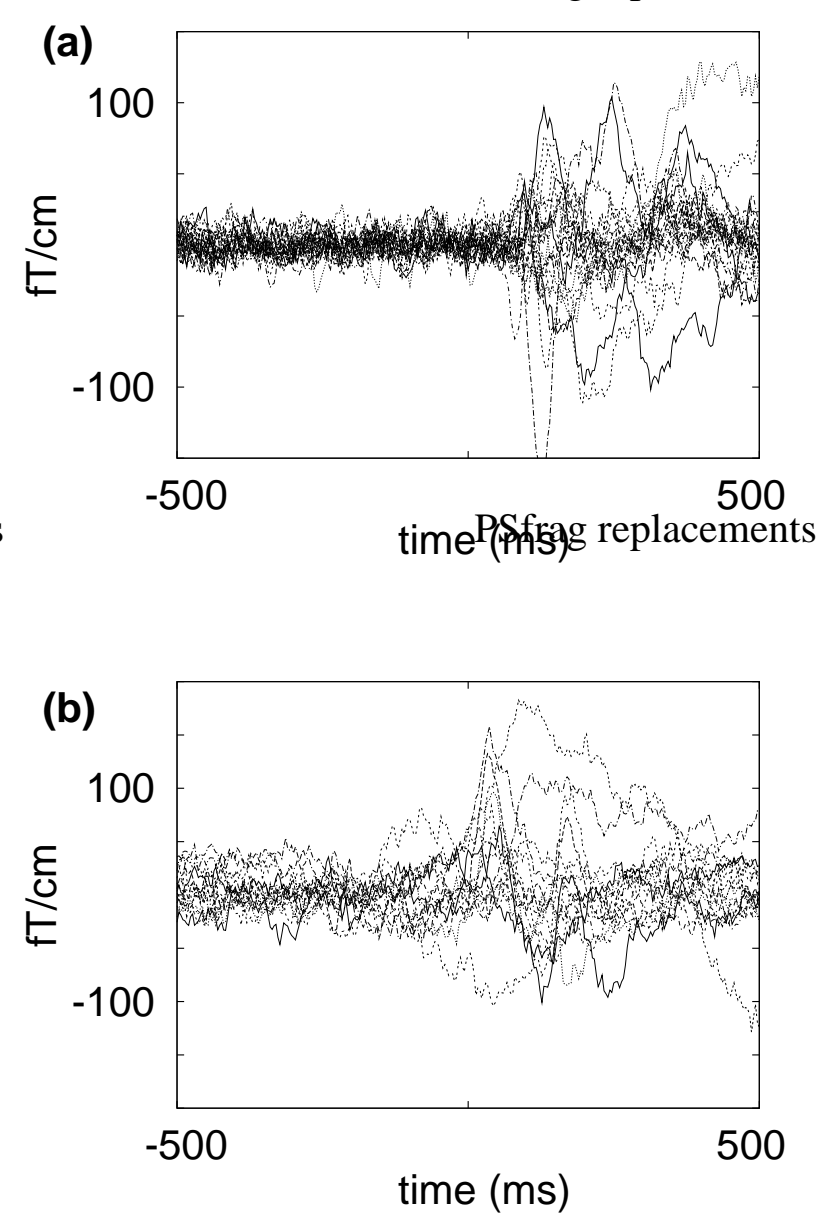
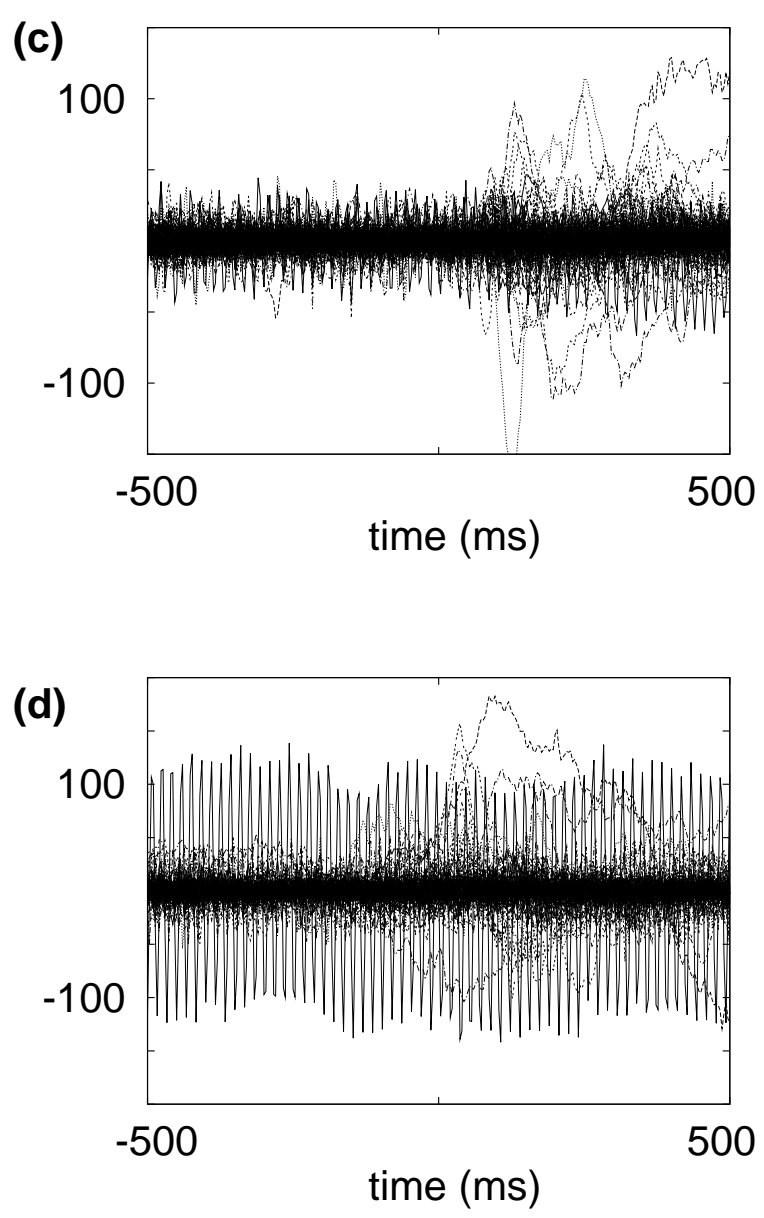

Figure 1: Event-triggered averages for groups of separated components ( $N=90$ trials). (a) Components showing visual-stimulus-triggered responses, triggered on visual stimulus onset. (b) Components showing button-press-triggered responses, triggered on button presses. (c) All components, triggered on visual stimulus onset. (d) All components, triggered on button presses.

the components (Table 1). The energies in the visual and somatosensory components (using visual stimuli and button presses as triggers respectively) were $10.0 \pm 1.02 \%(N=29)$ and $4.65 \pm 0.74 \%$ $(N=10)$. Using button presses as triggers (because subjects tended to blink after the button press responses), the energy in the ocular artifact components were $24.86 \pm 4.67 \%(N=16)$. Since both $60 \mathrm{~Hz}$ signals and sensor jumps were not task related, the energy in these two types of components were calculated using both visual stimulation and button presses as triggers and then averaged. The total energy in the $60 \mathrm{~Hz}$ sources was $10.19 \pm 1.73 \%(N=32)$ and the total energy in the sensor jump sources was $1.72 \pm 0.28 \%(N=13)$. The ocular artifact and $60 \mathrm{~Hz}$ components have the most energy, while the energy in the neuronal components represented $10 \%$ or less of the total. 


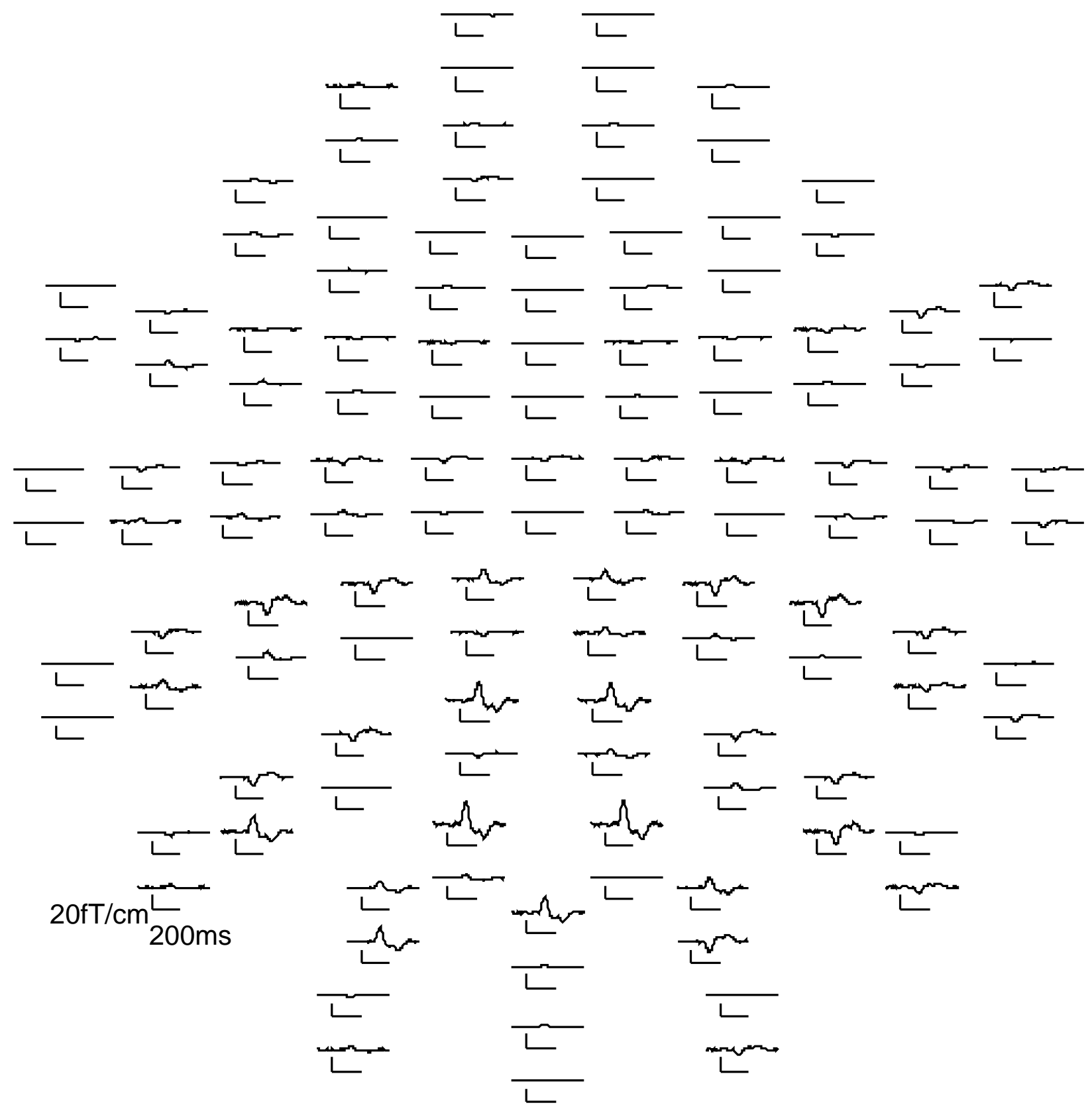

Figure 2: Sensor projection of component showing selective sensor activation over the occipitoparietal cortex ( $N=90$ trials, visual stimulus triggered averages). Compare with unseparated data in Fig. 4.

\subsection{Localization of separated components: examples}

Using the sensor projection of task-related components as input to standard Neuromag dipole fitting software (xfit), we localized separated components. In conventional source localization practice, very often only 20-30 channels are selected for source localization. To show how well SOBI can isolate one neuronal source from another without relying on channel exclusion, throughout 


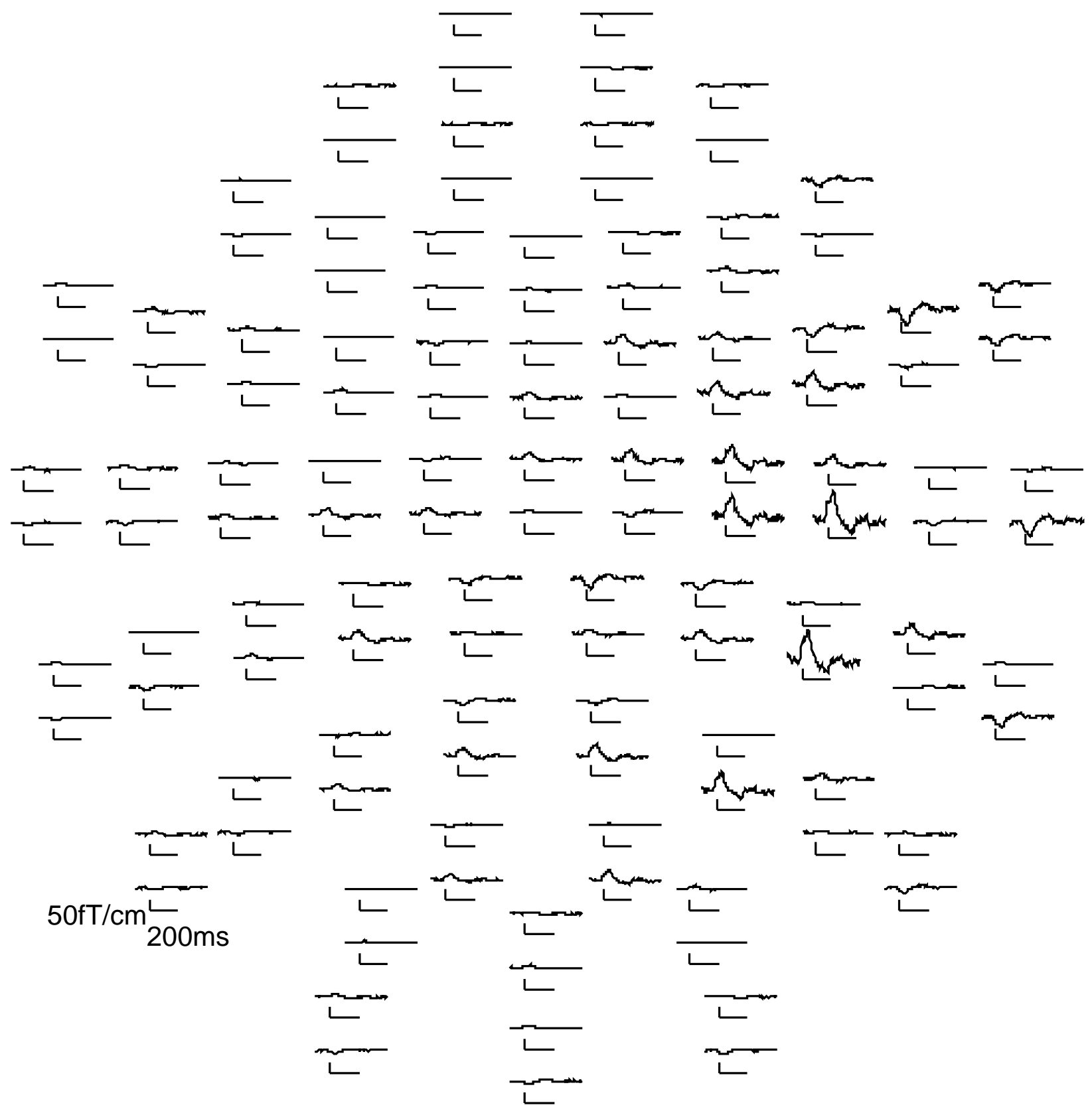

Figure 3: Sensor projection of component showing selective activation over the right frontoparietal cortex ( $N=90$ trials, button press triggered averages). Compare with unseparated data in Fig. 5.

Sec. 3.3-3.4, we generated the field maps, contour plots, and dipole localizations for components using all channels, i.e. without channel exclusion. To make the localization results comparable between using SOBI and without using SOBI, a subset of 20-30 channels were selected in dipole fitting in Sec. 3.5, which provides statistical comparisons. 


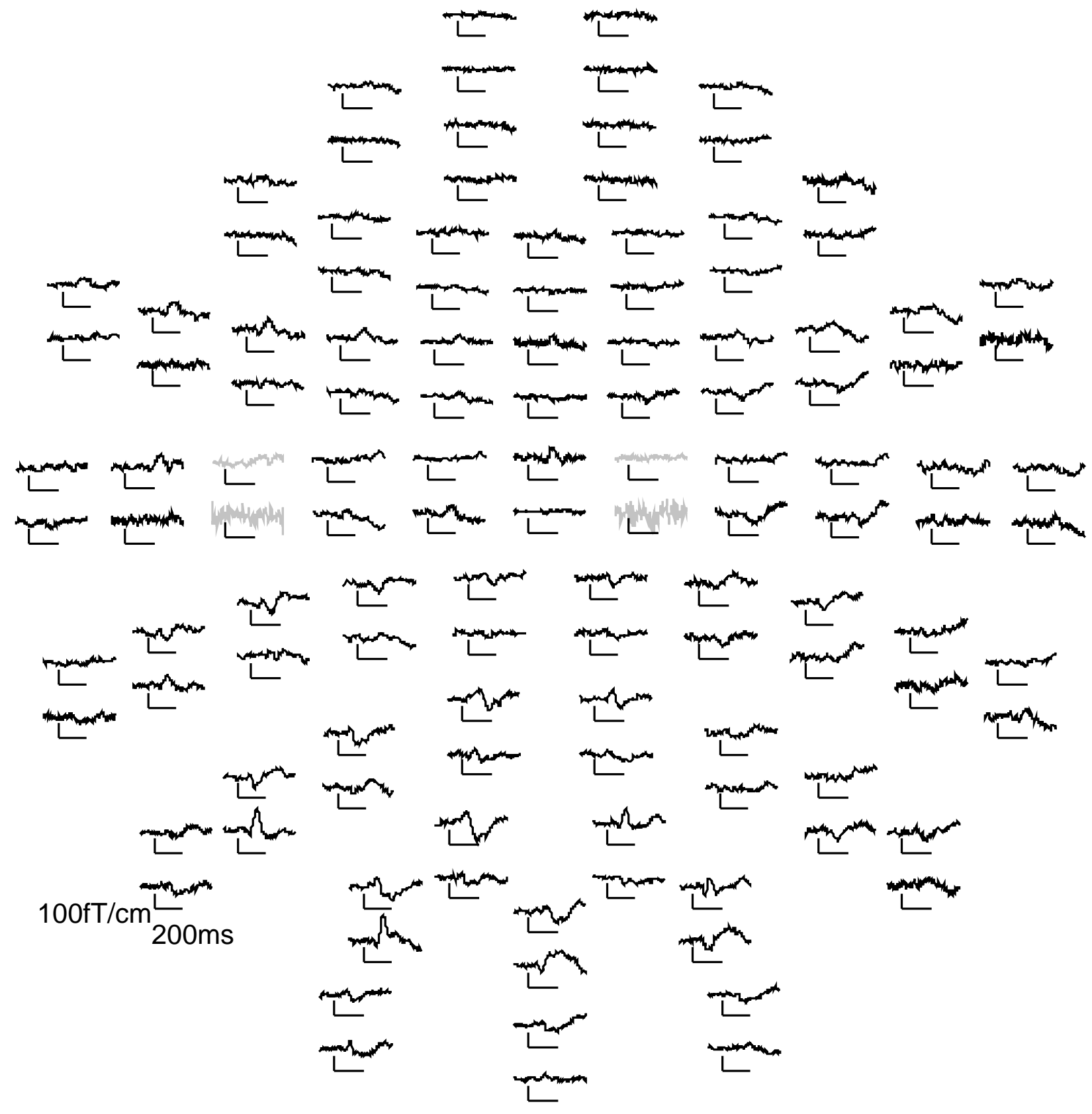

Figure 4: Visual stimulus triggered averages of unseparated data, $N=90$ trials. Aberrant sensors are shaded.

\subsubsection{Visual Component}

As the tasks involved simultaneous bilateral visual stimulation and judgment of its spatial location and identity, we expected SOBI to isolate visual components in the occipital, parietal, and temporal lobes. Components with visual evoked responses indeed showed field map activation over occipital, parietal and temporal lobes (not shown). For the particular stimuli used in these experiments, temporal sources were more variable in their precise location and temporal profiles. 


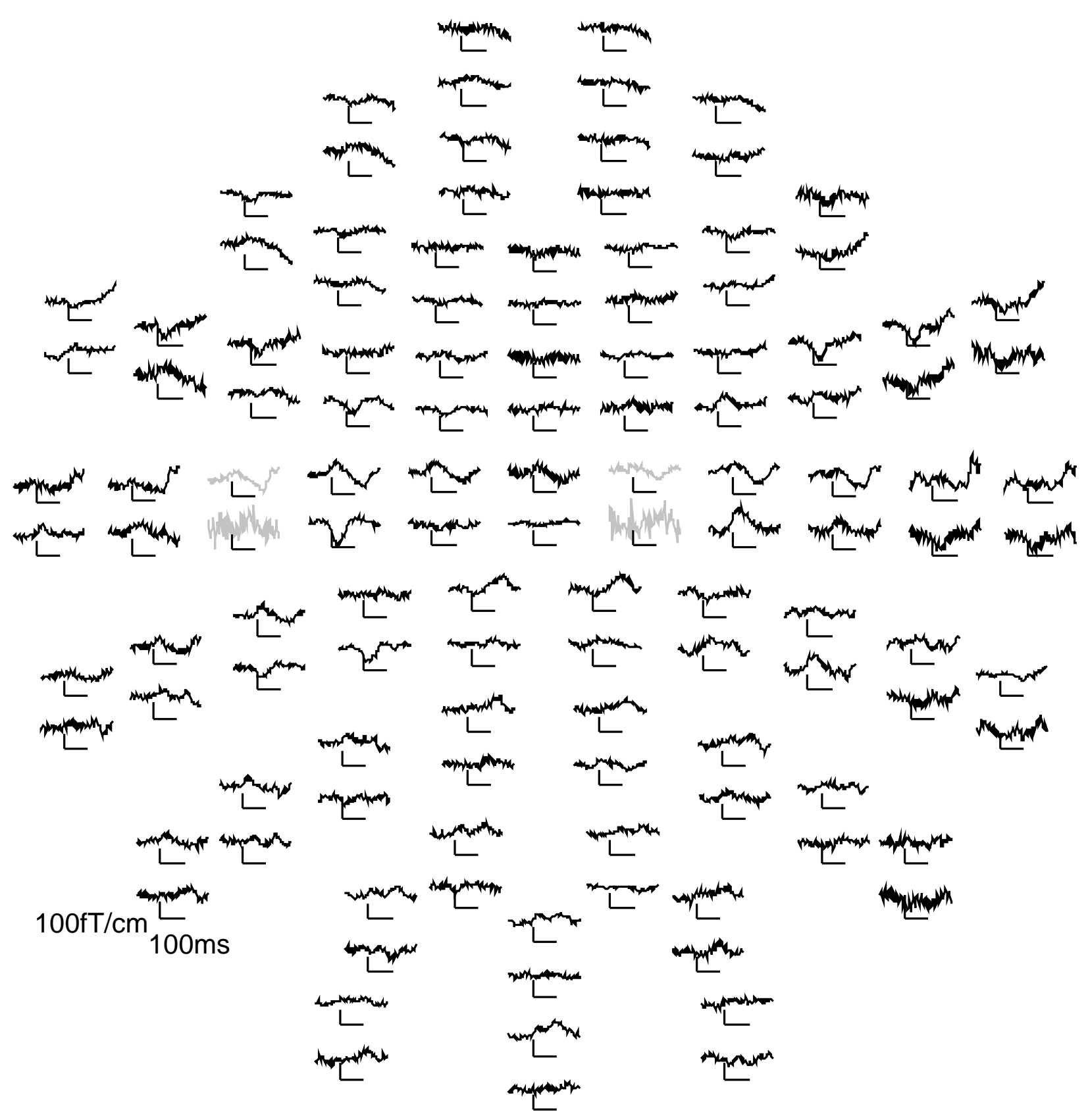

Figure 5: Button press triggered averages of unseparated data, $N=90$ trials. Aberrant sensors are shaded.

In contrast, occipito-parietal lobe activation appeared to have the greatest signal amplitudes and were reliably identified across multiple subjects. Fig. 7left shows the dipole location of one such occipito-parietal visual source along with its field map and time course. 

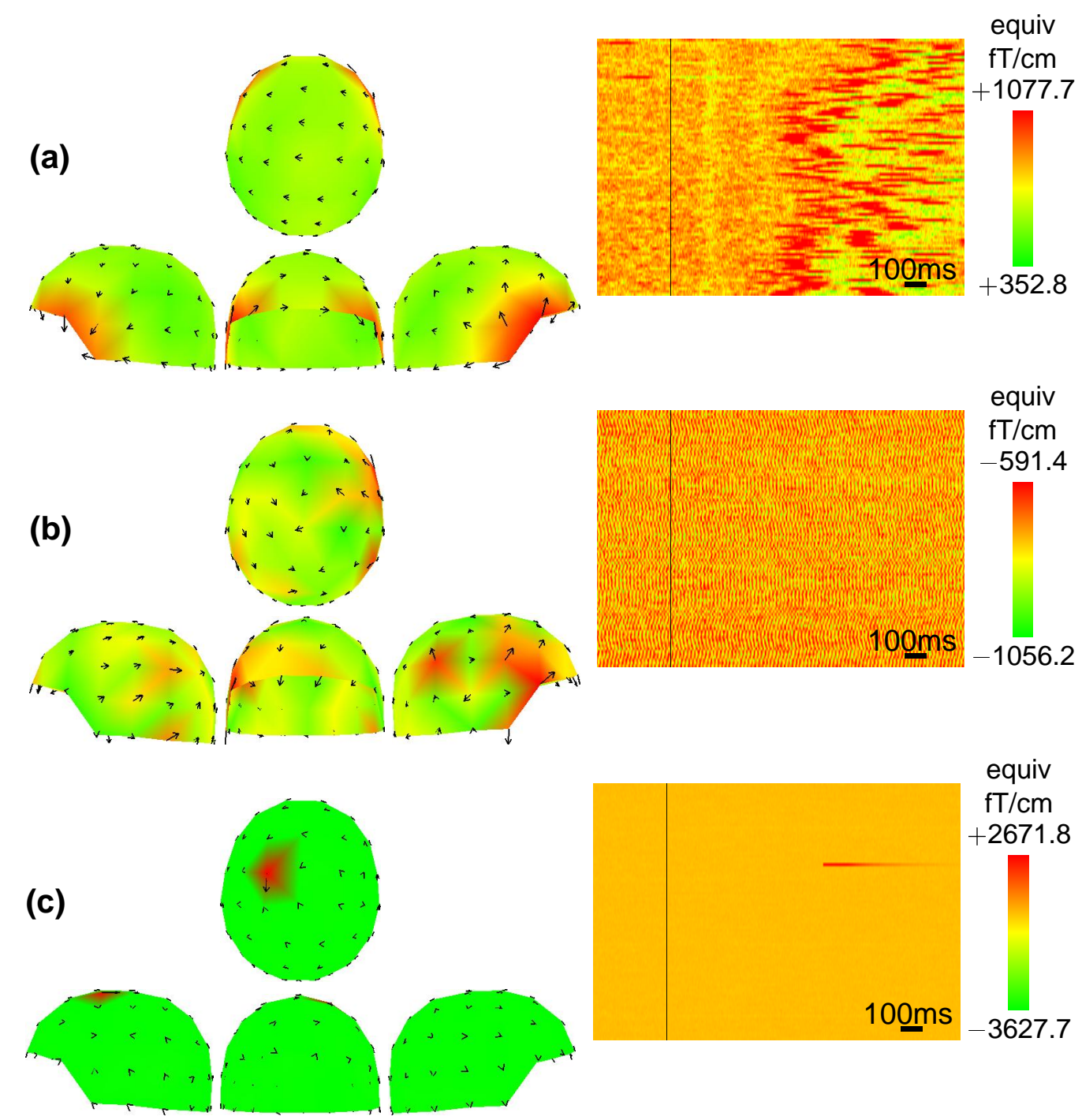

Figure 6: Field maps and unfiltered MEG images for (a) an ocular artifact component, (b) $60 \mathrm{~Hz}$ component, and (c) sensor jump component.

\begin{tabular}{ccc}
\hline Category & Minimum & Maximum \\
\hline Visual & 0.21 & 24 \\
Somatosensory & 0.47 & 14 \\
Ocular Artifact & 0.57 & 72 \\
60 Hz & 0.26 & 44 \\
Sensor Jump & 0.17 & 12 \\
\hline
\end{tabular}

Table 1: Range of energy accounted for (\% of total energy across all channels) by the five categories of components.

\subsubsection{Somatosensory Component}

As the tasks involved button presses, we expected both somatosensory and motor responses from the sensory and motor areas. Fig. 7middle shows the dipole location of one component in the 


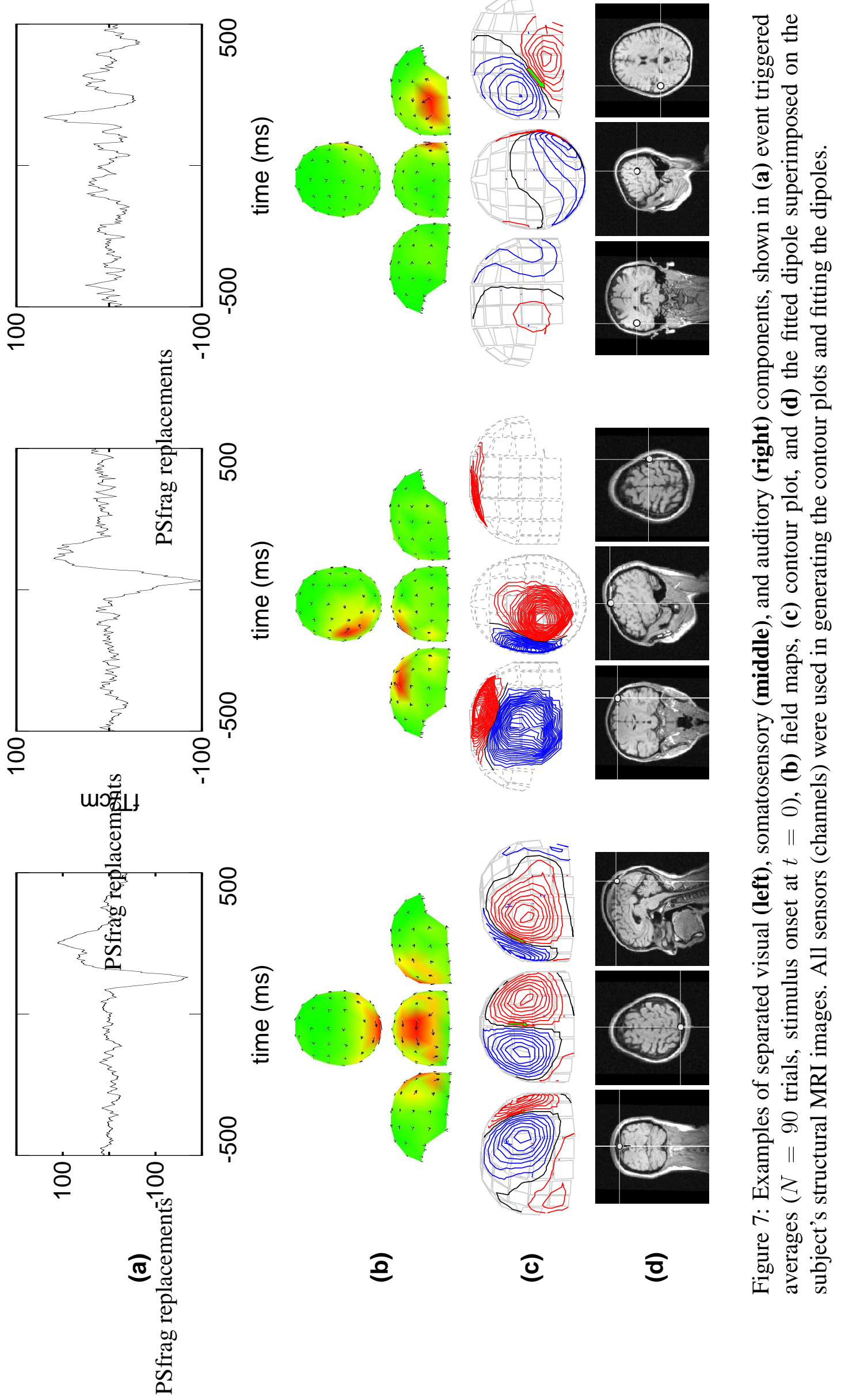


left hemisphere. Notice that this dipole is near the region where one finds dipoles from median nerve stimulation (Hari and Forss, 1999; Tesche and Karhu, 1997), and that the median nerve services the thumb. The time course of the response suggests that this response is unlikely to be a response from the motor cortex because the activations associated with motor preparation are typically estimated to be $385 \pm 85 \mathrm{~ms}$ before the movement onset (Hoshiyama et al., 1997), much earlier than the latency shown here. The motor-evoked sensory responses with an estimated onset time of approximately $20 \pm 30 \mathrm{~ms}$ after the onset of movement (Hoshiyama et al., 1997) matched best to our button-press-elicited responses. Therefore, this component corresponds to a somatosensory source instead of a motor source. In contrast to typical fast-rising somatosensory responses recorded using median nerve stimulation (Hari and Forss, 1999; Tesche and Karhu, 1997), the slow-rising somatosensory responses recorded here were elicited by stimulation to the thumb due to button presses. These temporal profiles are expected to differ, due to the difference between a very brief and focal electric shock and a much longer and distributed stimulation to the thumb and its surrounding areas.

\subsubsection{Auditory Component}

As the tones were presented as feedback, auditory responses were expected from the auditory cortex. In contrast to the typical auditory responses recorded during a simple auditory oddball task, the auditory responses from our experiment were most likely to overlap with and perhaps to be affected by both visual, motor, and somatosensory processing. As both auditory responses and somatosensory responses were triggered on the button press, auditory responses needed to be distinguished from the somatosensory sources. The spatial location of their fitted ECDs in the auditory cortex and their longer response latencies were sufficient to allow the disambiguation. Fig. 7right shows one unilateral auditory source, with slow-rising and long response latency, localized to the vicinity of the lateral fissure, as expected for auditory activation (Cansino et al., 1994).

The relatively longer response latency $(\sim 180 \mathrm{~ms})$ may be due to particular aspects of the task (see Appendix A). Specifically, in order to process the auditory feedback the subjects must first switch their attention from the visual to the auditory modality, and this takes time. Furthermore, the subjects must process and further interpret the auditory stimulus in evaluating their behavioral response and registering the correct target stimuli into memory. This additional processing may account for the difference in the temporal profile of the auditory responses. As the tones were bilaterally presented, one would expect auditory components with field maps showing bilateral activation. The auditory components recovered in these experiments, however, were unilateral for two subjects and bilateral for the other two. This variability across subjects could be due to differences in cerebral dominance of auditory processing. A difference in the temporal aspect of the left and right auditory processing could be expected to lead to the identification of two separate left and right components.

In comparison to visual and somatosensory components, auditory components were much more difficult to identify, perhaps due to the above described complexity and associated variability. Although in most cases auditory components could be identified from visual inspection of the field map and event-triggered averages, the signal-to-noise ratios were too poor to permit consistent dipole fitting across tasks and across subjects. Therefore, the following more detailed and systematic analysis of localization results will focus on only visual and somatosensory sources. 


\subsection{Cross-task and cross-subject reproducibility in localization of compo- nents}

To show how reproducible the localization of components can be across the four cognitive tasks, we examined separated visual components from one subject. Across tasks, two occipito-parietal visual sources were reliably localized within the same subject from two separated components . For both visual sources, the time course of the response is highly repeatable across multiple tasks, as shown in the overlay plot (Fig. 8a,b). The earlier visual responses were almost identical in both amplitude and response latency (Fig. 8a), while the later responses varied only in amplitude across tasks (Fig. 8b). Given the number of subjects in this study (four), we do not have the statistical power to draw any conclusions about whether the amplitude increases monotonically with the complexity of the task.

These visual components identified from different tasks were localized to similar locations within the occipital and parietal lobes, as shown in Fig. 8c,d, in which fitted dipoles from multiple experiments are superimposed on the subject's structural MRI images. Notice that in the field map, the right side of the head is shown on the right whereas in the structural MRI images, following radiological convention, the right side is shown on the left.

To show how reproducible the localization of components can be across subjects, we examined separated somatosensory components from three subjects. ${ }^{11}$ In all three subjects, we reliably identified two components (left and right) with button press locked responses in the somatosensory areas. Fig. 9 shows the time course, field map, contour plot, and fitted dipole for the somatosensory components in the right hemisphere of the three subjects. Notice the cross-subject similarity in the field maps, contour plots, and dipole locations (somatosensory cortex in the anterior parietal lobe, post-central sulcus).

\subsection{Detecting expected neuronal sources with and without SOBI}

To offer quantitative comparison in the relative performance of source localization with and without SOBI, we attempted to identify and localize the most reliable occipito-parietal visual source, and both the left and right somatosensory sources in all subjects and all tasks from separated components and from the unprocessed data. As all four tasks involved bilateral presentation of visual stimuli, we expected that at least one visual source would be found active in the occipito-parietal cortex. Similarly, because separate left and right button presses were required by all the tasks, we also expected that at least one left and one right somatosensory source would be active. For these expected sources, we attempted to localize the source with dipole fitting from separated components and from the raw sensor data (without SOBI). The percentage of the expected sources for which dipole solutions can be found are compared for localization with and without the aid of SOBI.

For a component to be considered a detectable neuronal source, there must be an evoked response that clearly deviates from the baseline in the averaged component data. We rejected all components with any ambiguity on this criterion. Secondly, the components must have a field map showing focal activation of sensors over the relevant brain regions (occipito-parietal cortex and

\footnotetext{
${ }^{11}$ The fourth subject did right-hand index-mid finger button presses which differed from the rest of the subjects.
} 
g replacements

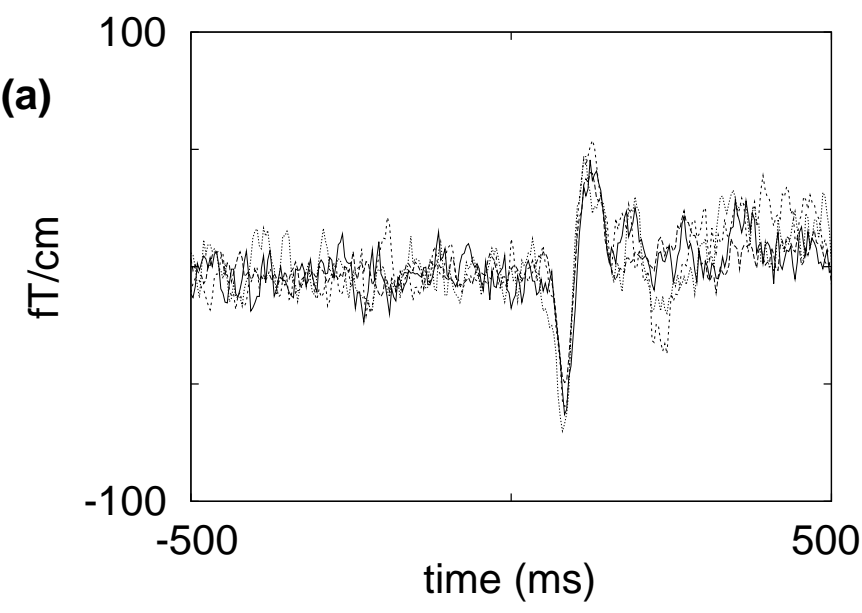

(c)
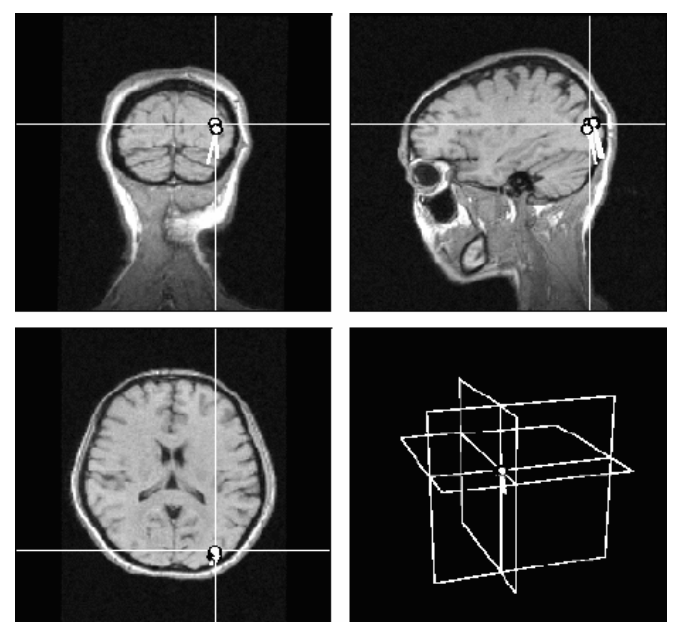

(b)

g replacements

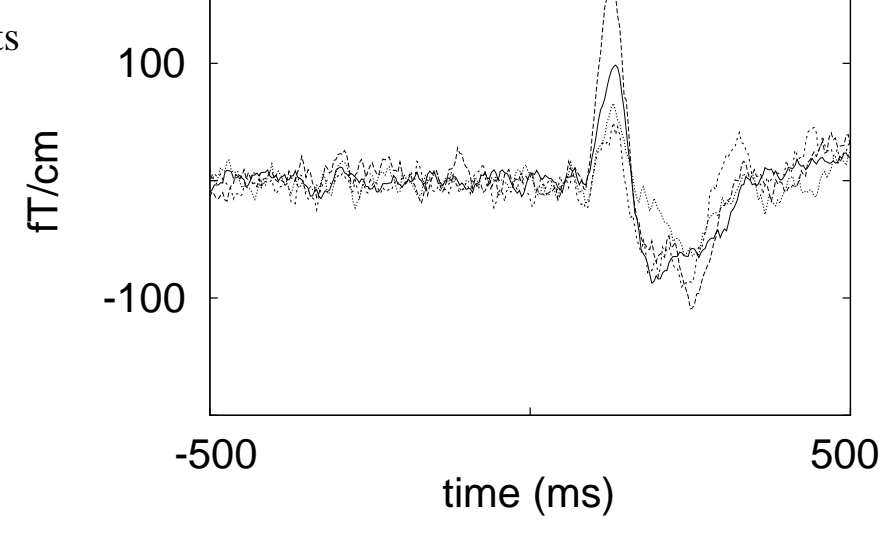

(d)
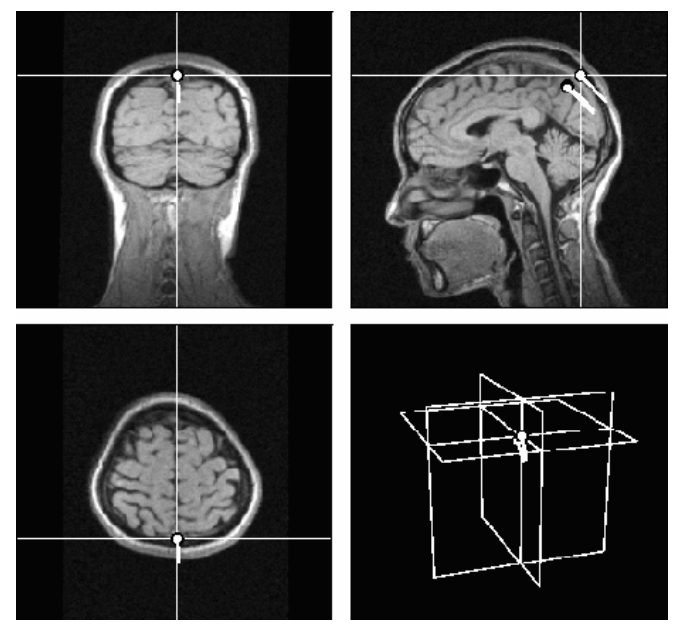

Figure 8: Cross-task consistency in the temporal profile (ab) and dipole location (cd) of two visual components. Occipital (ac) and occipito-parietal (bd) sources can be identified and localized consistently across multiple tasks (overlay). (ab) Visual stimulus-triggered averages from 4 visual tasks, overlayed ( $N=90$ trials per task). (cd) Corresponding single ECDs for visual sources in (ab). Notice consistency of the dipole locations across-tasks. Notice also the temporal profile of the earlier visual source (a) did not differ across tasks, but the amplitude of the later visual source (c) was modulated by the task conditions.

anterior parietal cortex in this study). Thirdly, the contour plot for the component must be dipolar. Finally, the fitted dipole must be in the relevant cortical areas. For a source to be considered detectable using the conventional method of localization, one must first identify a sensor at which the largest evoked response is found. Secondly, the contour plot must be dipolar at the peak time. Finally, in a few cases when multiple dipole solutions are needed, at least one of the dipoles is localized to the expected brain region. 


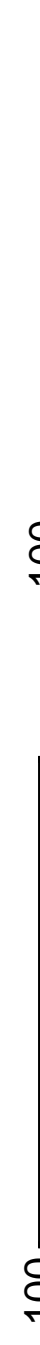

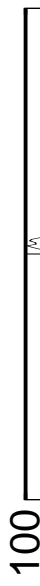
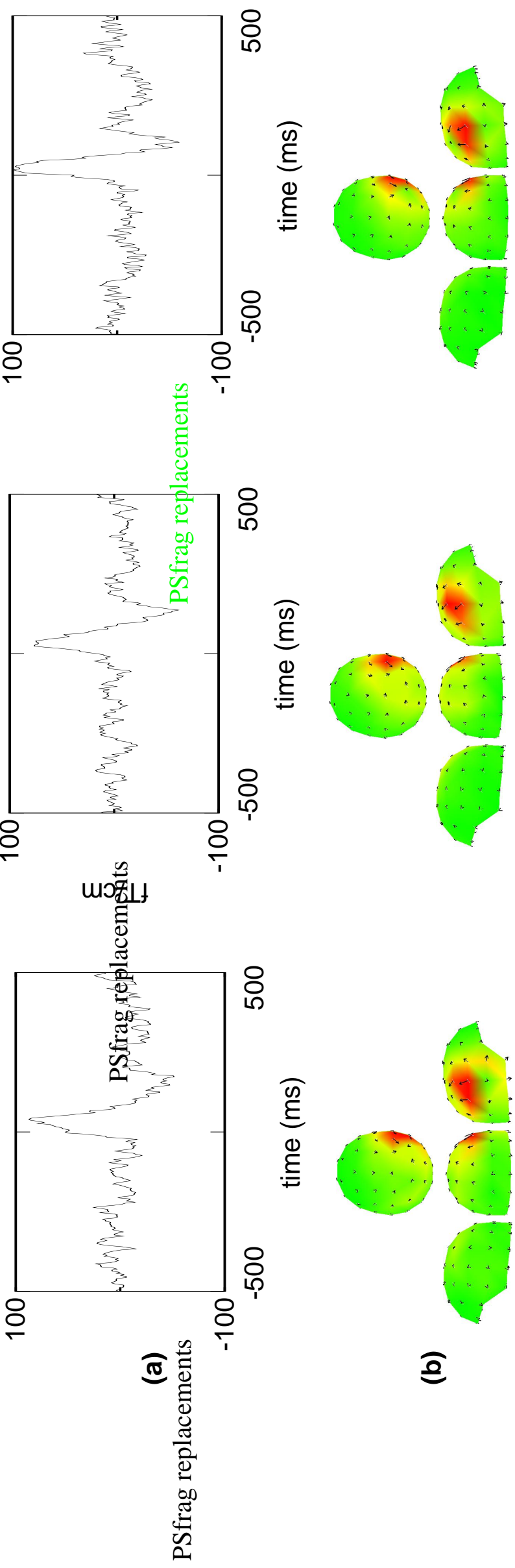
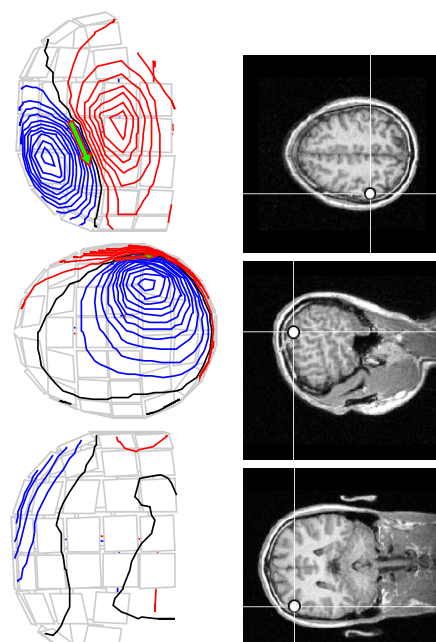

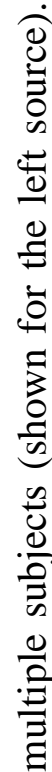
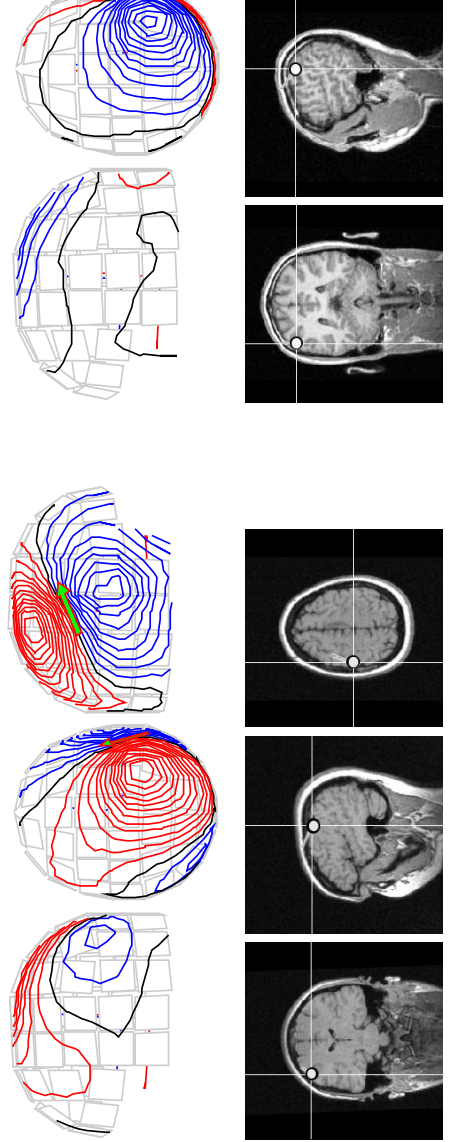

dั

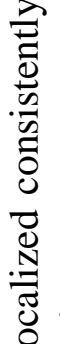

으

흥

류
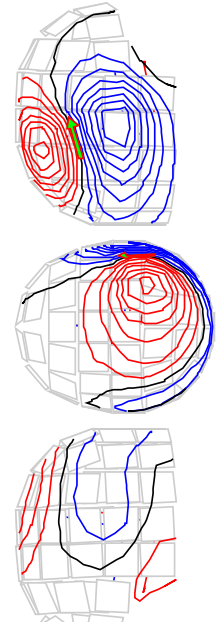

อ

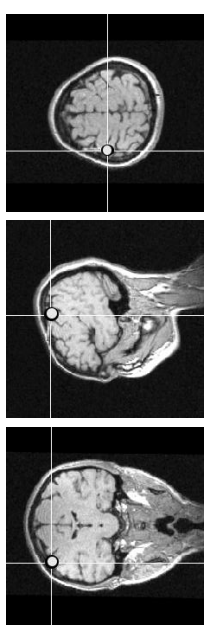

흐

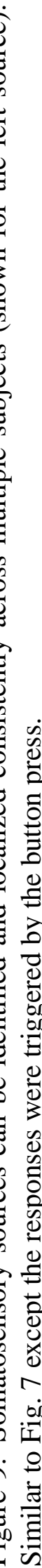




\subsubsection{Visual sources}

Among all separated components, for each subject and each task, we were able to identify and localize an occipito-parietal visual source with a single dipole (100\% detectability). These occipitoparietal components invariantly had very focal sensor projections (see field maps), and the contour plots were invariably dipolar even without channel selection (for example, see field map and contour plot in Fig. 7a). Single dipoles were fitted for these occipito-parietal components. A subset of channels over the occipito-parietal lobe (20-30 channels) were used for the purpose of fair comparison with the conventional analysis method without the aid of SOBI. The peak response latencies of these components $(N=16)$ were $139.0 \pm 7.6$ and the dipole coordinates $(\mathrm{X}, \mathrm{Y}, \mathrm{Z})$ were $7.5 \pm 2.6$, $-49.4 \pm 3.2$, and $68.6 \pm 3.4 \mathrm{~mm}$.

Using the conventional method of source localization directly from the unseparated sensor data, dipoles were fitted using the same or similar subset of channels selected over the occipitoparietal cortex. In all subjects and all tasks, the conventional method identified and localized at least one visual source in the occipito-parietal lobe (100\% detectability). Of a total of 16 expected sources ( 4 tasks by 4 subjects by 2 sides), 10 could be fitted with a single dipole, 4 were fitted with two-dipole solutions, 1 was fitted with a three-dipole solution, and 1 was fitted with a four-dipole solution. When multiple dipole solutions were needed, at least one of them was localized to the occipito-parietal cortex. This variation in dipole solutions may reflect some individual differences in visual processing occurring outside of the occipito-parietal cortex. The peak response latencies of these occipito-parietal visual sources $(N=16)$ were $143.6 \pm 5.5$ and the dipole coordinates $(\mathrm{X}, \mathrm{Y}, \mathrm{Z})$ were $4.21 \pm 4.8,-55.89 \pm 2.68$, and $59.42 \pm 3.83 \mathrm{~mm}$.

\subsubsection{Somatosensory source}

From components of all subjects and all tasks, with only two failures we were able to identify and localize 22 out of the 24 expected left and right somatosensory sources with a single dipole (3 subjects by 4 tasks). All 22 somatosensory components had very focal sensor projections (see field maps) and their contour plots were all highly dipolar even without channel selection (for example, see field map and contour plot in Fig. 9.) Single dipoles were fitted to these components, with a subset of channels over the somatosensory cortex (20-30 channels) selected for the purpose of fair comparison with the conventional analysis method. The peak response latencies were $33.3 \pm 4.2$ and $30.8 \pm 3.4 \mathrm{~ms}$ for the left $(N=11)$ and right $(N=11)$ somatosensory sources. The dipole coordinates $(\mathrm{X}, \mathrm{Y}, \mathrm{Z})$ were $-39.4 \pm 2.4,7.8 \pm 2.7$, and $84.6 \pm 1.7$ for the left and $45.69 \pm 2.1,5.6 \pm 2.2$, and $84.1 \pm 3.1$ for the right somatosensory sources.

Using the conventional method of source localization directly from the unseparated sensor data, dipoles were fitted using the same or similar subset of channels selected over the somatosensory cortex. Only 9 out of 24 expected left and right somatosensory sources could be identified and localized following the conventional method of identifying a peak response in the averaged sensor data. Of 24 sources expected ( 3 subjects by 4 tasks by 2 sides), in 7 cases no visible peak response could be identified in any of the sensors. Of the remaining 17 cases in which peak responses could be found in at least one sensor over the somatosensory cortex, 4 did not have dipolar fields, and 4 resulted in dipole locations outside of the head or in the auditory cortex. Single dipole solutions were found in only 9 cases. The peak response latencies of these somatosensory sources were $24.8 \pm 2.5$ for the left hemisphere $(N=5)$ and $31.6 \pm 1.8$ for the right hemisphere $(N=4)$. 

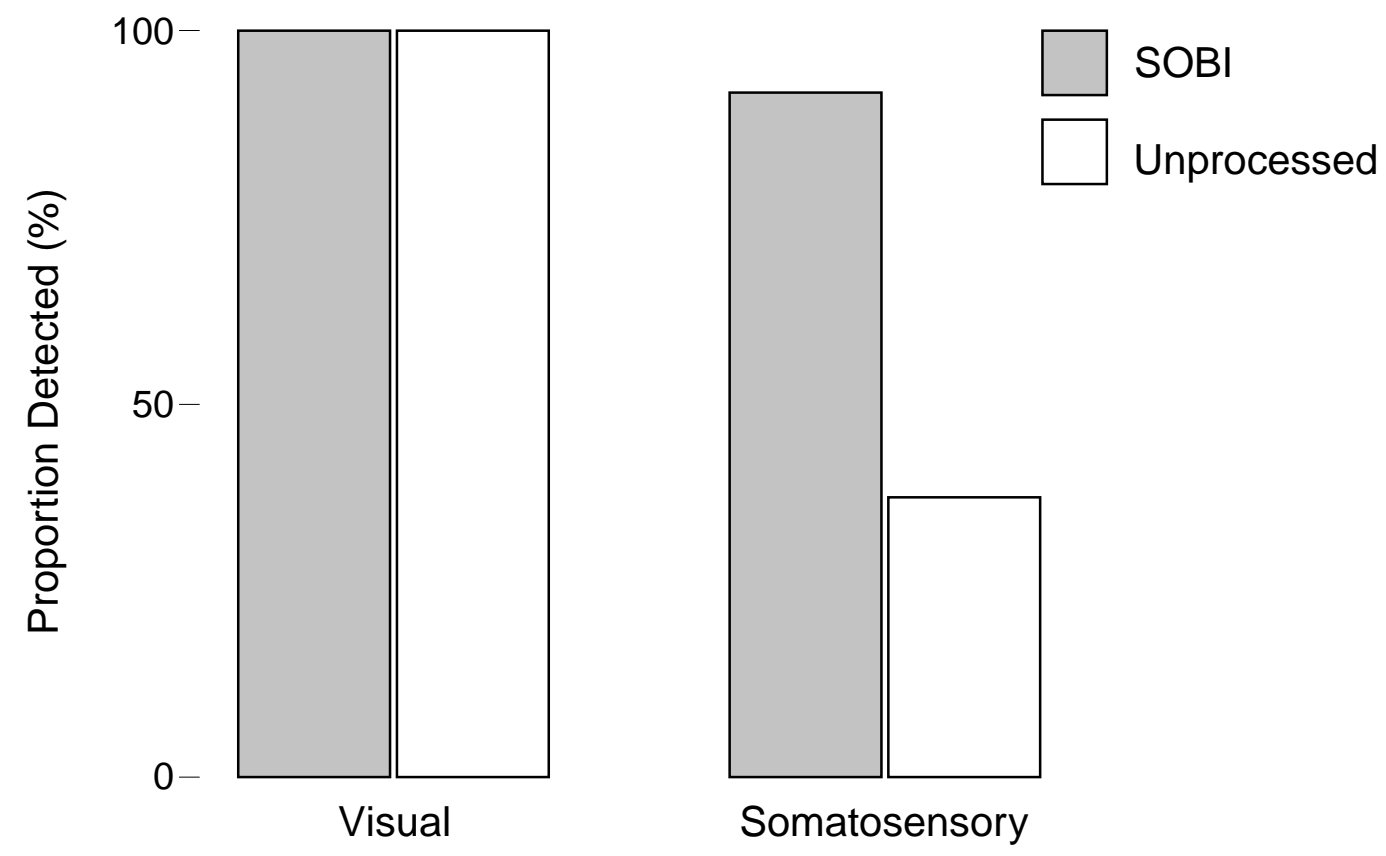

Figure 10: SOBI increased the detectability of expected neuronal sources for the more variable somatosensory activation.

The dipole coordinates $(\mathrm{X}, \mathrm{Y}, \mathrm{Z})$ were $-43.3 \pm 3.9,12.1 \pm 5.6$, and $82.8 \pm 3.8$ for the left $42.3 \pm 5.5$, $15.9 \pm 2.7$ and $89.9 \pm 1.4$ for the right sources.

\subsection{Statistical comparisons}

There was no significant difference in the detectability for the occipito-parietal source measured with and without SOBI. In contrast, SOBI preprocessing resulted in an increase in the detectability of the expected somatosensory sources (Chi Squre, test $p<$.0001) (Fig. 10). The peak response latencies for the visual and somatosensory sources did not differ significantly when measured using and without using SOBI. For the visual sources, the precise dipole locations estimated with and without SOBI did not differ in the $\mathrm{X}$ and $\mathrm{Y}$ dimensions but nearly differed significantly in the $\mathrm{Z}$ dimension $(p=0.05)$. For the somatosensory sources, the precise dipole locations differed significantly in the $\mathrm{Y}$ dimension $(p<0.05)$ for the left source and in $\mathrm{Y}$ and $\mathrm{Z}$ dimensions for the right source $(p<0.05)$. As the true accuracy of source locations cannot be determined from these experiments without a depth-electrode, no quantitative comparisons can be made concerning accuracy.

\section{Discussion}

We identified and localized visual and somatosensory sources activated in four subjects during four cognitive tasks. Due to the relatively large variability involved in highly cognitive tasks and the small number of trials collected, these tasks were characterized by relatively poor signal-tonoise ratios in the sensor data and therefore were ideal for evaluating differential localization per- 
formance. Our results showed that despite the large variability associated with the visual and somatosensory activations during these particular tasks, SOBI was able to separate identifiable visual and somatosensory components that were further localized to the expected cortical regions. The physiological and neuroanatomical interpretability of these components across multiple sensory modalities and their cross-subject and cross-tasks reproducibility establishes SOBI as a viable method for separating and identifying neuronal populations from MEG data during fairly complex cognitive tasks. Most importantly, we showed that SOBI preprocessing offered a special advantage when the evoked responses in the sensor data had poor signal-to-noise ratios. Specifically, for the highly variable somatosensory activation evoked by incidental stimulation during button presses, SOBI preprocessing resulted in a greater percentage of the expected somatosensory sources being identified and localized than the same dipole modeling method applied directly to the raw sensor data.

\subsection{SOBI reduced subjectivity and labor in source localization}

In conventional source localization, there are two major sources of subjectivity: the selection of dipole fitting times, and the selection of channels. These are both eliminated by our proposed procedure. First, because each component has a fixed field map, the dipole fitting solutions for components were not sensitive to either the time at which the dipoles were fitted nor to the sensor used for determining the time of fit (see Sec. B.2). Within this map, each sensor reading reflects only activation due to a single source generator, or several temporally coherent generators as opposed to activation due to a combination of multiple generators, each with a different time course. Therefore, using SOBI, there is no need to subjectively select a time from a sensor for dipole fitting. Secondly, simple components, which have field activation over early sensory processing areas, were almost always dipolar even without channel selection/reduction. ${ }^{12}$ Therefore, channel selection is not necessary. One way to see the difference between dipole localization with and without SOBI processing is to view SOBI as a more automatic and more objective tool that allows the isolation of sensor activation due to an already isolated functionally independent generator. The reduced subjectivity and time required to find dipole solutions can make data analysis and training of new researchers for MEG more cost-effective.

\subsection{SOBI improved detectability of neuronal sources}

The advantages of ICA algorithms in general have been shown in a number of applications to EEG and MEG data. First, these algorithms can separate neuronal activity from various artifacts (Makeig et al., 1996; Vigário et al., 1998; Tang et al., 2000a; Ziehe et al., 2000; Jung et al., 2000a,b), such as eye blinks. In contrast to methods that rely on the use of a template, ICA removes these artifacts without any prior assumptions about the nature of the waveforms. Secondly, ICA isolates physiologically and behaviorally meaningful components that describe previously unavailable aspects of neuronal activity (Makeig et al., 1997, 1999b; Wübbeler et al., 2000). Finally,

\footnotetext{
${ }^{12} \mathrm{SOBI}$ also separated out many complex components which have multiple patches or very broad field activation. These components may reflect synchronized activation in multiple brain regions. Functional connectivity may be inferred among these brain regions.
} 
ICA-separated neuronal sources are less contaminated by various noise sources, which allows single-trial response detection (Jung et al., 1999; Tang et al., 2000b; Carter et al., 2000). ICA methods have been able to distinguish the absence of rhythmic activity from the absence of phase locked rhythmic activity (Makeig et al., 1999a).

We have shown that SOBI separation of the data resulted in a greater detectability of somatosensory sources, but did not increase the detectability of visual sources. This modality-specific improvement in source detectability depended on the signal-to-noise ratios in the sensor data. Because visual responses could be clearly identified from the raw sensor data even without the aid of SOBI, it would not have been possible for SOBI to improve the detection rate. In contrast, the relatively poor signal-to-noise ratios in the raw sensor data for the somatosensory responses caused many failures in identifying a sensor at which a peak response occurred and in determining the peak response time. Under this poor signal-to-noise condition, in all but two cases, SOBI preprocessing resulted in separated components with the characteristic field map, characteristic temporal response profile, and the correct dipole location for a somatosensory source. These findings suggest another advantage that ICA algorithms can offer: improving the ability to detect and localize neuronal sources that are otherwise difficult to detect or are undetectable under relatively poor signal-to-noise conditions.

This improvement has significant practical implications. First, brain regions involved in higher level cognitive processing tend to show greater trial-to-trial variability in their activation, and therefore, have lower signal-to-noise ratios in the average responses. Second, behavioral tasks that bear greater resemblance to real world situations tend to involve greater variability in both stimulus presentation and subsequent processing. Finally, studies of clinical patients and children are often limited by the length of the experiment, and therefore, often provide data from a limited number of trials. Our results suggest that ICA may offer an improved capability in detecting and localizing neuronal source activations in these difficult situations.

\subsection{Assumptions of SOBI}

Here, we discuss assumptions of particular relevance to SOBI and MEG, rather than general issues in ICA. Like all ICA algorithms, SOBI assumes that the mixing process is stable. In the context of MEG, a stable mixing process corresponds to assuming that the head is motionless relative to the sensors. For this reason head stabilization can be particularly important in MEG when ICA is used. SOBI also assumes that there are at least as many sensors as sources. For us, this is not a serious problem, as our MEG device has 122 sensors, yet we recover only a few dozen sources that show task-related evoked responses. The observation that only a small number of sources are active during typical cognitive and sensory activation tasks is consistent with the results of studies using both EEG (Makeig et al., 1999b) and MEG Vigário et al. (2000). The crucial assumption in ICA is that of independence. For a thorough discussion of the independence assumption as it pertains to MEG, see Vigário et al. (2000). Here, we will discuss independence only in the context of the particular measure of independence used by SOBI.

One problem that EEG and MEG researchers have with the independence assumption arises from the fact that if one computes correlations between EEG or MEG sensor readings over multiple brain regions during behavioral tasks, one would find that some brain regions have non-zero correlations. A good example of correlated brain activity is the apparently correlated evoked responses 
from neuronal populations in multiple visual areas along the processing pathway during a visual stimulus presentation. Based on such an observation, one could conclude that as the statistical independence assumed by ICA is clearly violated, the results of ICA must not be trusted. Yet, we have shown that SOBI was able to separate visual components that clearly correspond to neuronal responses from early and later visual processing stages that are correlated due to common input (Tang et al., 2000b). Others (Makeig et al., 1999b; Vigário et al., 2000) have produced behaviorally and neurophysiologically meaningful components under a variety of task conditions.

As different ICA algorithms use the independence assumption differently, we offer the following explanation that applies specifically to SOBI. One needs to recognize that correlation is not a binary quantity. Consequently, neither is violation of the independence assumption. The important question is not whether the assumption is violated but whether the assumption is sufficiently violated such that the estimated neuronal sources by SOBI are no longer meaningful. The way SOBI uses the independence assumption is to minimize the total correlations computed with a set of time delays, as described in Appendix B.1. As such, each delay-correlation matrix $\mathbf{R}_{\tau}$ generally makes only a small contribution to the objective function. For example, the correlation one would observe between $\mathrm{V} 1$ and $\mathrm{V} 2$ responses could be high only at or around one particular time delay, say in $\mathbf{R}_{20 \mathrm{~ms}}$. In optimizing its objective function, $\mathrm{SOBI}$ can leave a particularly large non-zero off-diagonal element, say the one corresponding to the $20 \mathrm{~ms}$ delayed correlation between $\mathrm{V} 1$ and $\mathrm{V} 2$, when minimizing; the sum squared off-diagonal elements across all the components and time delays. Therefore, this particular method of maximizing independence is not necessarily incompatible with a large correlation at a particular time delay between two sources sharing common inputs.

Most ICA algorithms, including SOBI, minimize some objective function. It is possible for the optimization process to find a poor local minimum. In general, poor results can result from many underlying causes: poor experimental design, poorly conducted experiments, poor head stabilization, poor optimization within the ICA algorithm, violation of assumptions, etc. No amount of attention to any one possible problem can validate ICA-based methods for processing functional brain imaging data. As with any statistical procedure, the real issue here should not be whether assumptions are violated at all, but whether the algorithms can robustly produce separated components that are behaviorally, neuroanatomically, and physiologically interpretable, despite some violation of the assumptions under which the algorithms were derived. For example, t-tests are very robust against the violation of normality assumption and are therefore regularly performed on data which are not guaranteed to be Gaussian. Only empirical results can give confidence that a method is correctly separating the MEG data.

\subsection{Summary}

Establishing that (1) SOBI preprocessing can lead to the identification and localization of physiologically and anatomically meaningful neuronal sources and (2) SOBI preprocessing can increase the success rate in detecting and localizing neuronal source activation under poor signal-to-noise conditions is only the first step in demonstrating the usefulness of ICA algorithms to the analysis and interpretation of MEG data. The next steps include systematically studying the effect of ICA on source localization when ICA methods are combined with more sophisticated source localization algorithms (Ribary et al., 1991; Aine et al., 1998; Mosher and Leahy, 1999; Schmidt et al., 
1999) and exploring the possibility of measuring single-trial response onset times in ICA separated neuronal sources.

\section{Acknowledgements}

Supported by the National Foundation for Functional Brain Imaging and NSF CAREER award (9702-311), an equipment grant from Intel corporation, the Albuquerque High Performance Computing Center, a gift from George Cowan, and a gift from the NEC Research Institute. We thank Ole Jensen for tips on packaging the separated components for the Neuromag software, Mike Weisend for granting us access to his data, and Robert Christner for technical support. We also thank Lloyd Kaufman, Zhonglin Liu, Claudia Tesche, Cheryl Aine, and Roland Lee for their comments and discussion.

\section{References}

Aine, C., Huang, M., Stephen, J., and Christner, R. (2000). Multistart algorithms for MEG empirical data analysis reliably characterize locations and time courses of multiple sources. Neuroimage, 12(2):159-172.

Aine, C. J., Huang, M., Christner, R., Stephen, J., Meyer, J., Silveri, J., and Weisend, M. (1998). New developments in source localization algorithms: Clinical examples. International Journal of Psychophysiology, 30:198.

Amari, S.-I. and Cichocki, A. (1998). Adaptive blind signal processing-neural network approaches. Proceedings of the IEEE, 9.

Bell, A. J. and Sejnowski, T. J. (1995). An information-maximization approach to blind separation and blind deconvolution. Neural Computation, 7(6):1129-1159.

Belouchrani, A., Meraim, K. A., Cardoso, J.-F., and Moulines, E. (1993). Second-order blind separation of correlated sources. In Proc. Int. Conf. on Digital Sig. Proc., pages 346-351, Cyprus.

Cansino, S., Williamson, S. J., and Karron, D. (1994). Tonotopic organization of human auditory association cortex. Brain Res., 663:38-50.

Cao, J. T., Murata, N., Amari, S., Cichocki, A., Takeda, T., Endo, H., and Harada, N. (2000). Single-trial magnetoencephalographic data decomposition and localization based on independent component analysis approach. IEICE Transactions on Fundamentals of Electronics, Communications and Computer Sciences, E83A(9):1757-1766.

Cardoso, J.-F. (1994). On the performance of orthogonal source separation algorithms. In European Signal Processing Conference, pages 776-779, Edinburgh.

Cardoso, J.-F. (1998). Blind signal separation: statistical principles. Proceedings of the IEEE, 9(10):2009-2025.

Cardoso, J.-F. and Souloumiac, A. (1996). Jacobi angles for simultaneous diagonalization. SIAM Journal of Matrix Analysis and Applications, 17(1). 
Carter, S. A., Tang, A. C., Pearlmutter, B. A., Anderson, L. K., Aine, C. J., and Christner, R. (2000). Coactivation of visual and auditory pathways induces changes in the timing of evoked responses in populations of neurons: an MEG study. Society for Neuroscience Abstracts, 26(9197).

Ermer, J. J., Mosher, J. C., Huang, M. X., and Leahy, R. M. (2000). Paired MEG data set source localization using recursively applied and projected (RAP) MUSIC. IEEE Transactions on Biomedical Engineering, 47(9):1248-1260.

George, J. S., Aine, C. J., Mosher, J. C., Schmidt, D. M., Ranken, D. M., Schlitt, H. A., Wood, C. C., Lewine, J. D., Sanders, J. A., and Belliveau, J. W. (1995). Mapping function in the human brain with magnetoencephalography, anatomical magnetic resonance imaging, and functional magnetic resonance imaging. J. Clin. Neurophysiol., 12:406-431.

Hamalainen, M., Hari, R., Ilmoniemi, R. J., Knuutila, J., and Lounasmaa, O. V. (1993). Magnetoencephalography - theory, instrumentation, and applications to noninvasive studies of the working human brain. Rev. Modern Physics, 65:413-497.

Hari, R. and Forss, N. (1999). Magnetoencephalography in the study of human somatosensory cortical processing. Phil. Trans. R. Soc. Lond. B, 354:1145-1154.

Hari, R. and Salmelin, R. (1997). Human cortical oscillations: a neuromagnetic view through the skull. Trends in Neuroscience, 20:44-49.

Hoshiyama, M., Kakigi, R., Berg, P., Koyama, S., Kitamura, Y., Shimojo, M., Watanabe, S., and Nakamura, A. (1997). Identification of motor and sensory brain activities during unilateral finger movement: Spatiotemporal source analysis of movement-associated magnetic fields. Exp. Brain Res., 115(1):6-14.

Huang, M. X., Aine, C., Davis, L., Butman, J., Christner, R., Weisend, M., Stephen, J., Meyer, J., Silveri, J., Herman, M., and Lee, R. R. (2000). Sources on the anterior and posterior banks of the central sulcus identified from magnetic somatosensory evoked responses using multi-start spatio-temporal localization. Human Brain Mapping, 11(2):59-76.

Hyvärinen, A. (1999). Survey on independent component analysis. Neural Computing Surveys, 2:94-128.

Hyvärinen, A. and Oja, E. (1997). A fast fixed-point algorithm for independent component analysis. Neural Computation, 9(7).

Jung, T.-P., Humphries, C., Lee, T.-W., McKeown, M. J., Iragui, V., Makeig, S., and Sejnowski, T. J. (2000a). Removing electroencephalographic artifacts by blind source separation. Psychophysiology, 37:163-178.

Jung, T.-P., Makeig, S., Westerfield, M., Townsend, J., Courchesne, E., and Sejnowski, T. J. (1999). Analyzing and visualizing single-trial event-related potentials. In Advances in Neural Information Processing Systems 11, pages 118-124. MIT Press.

Jung, T.-P., Makeig, S., Westerfield, M., Townsend, J., Courchesne, E., and Sejnowski, T. J. (2000b). Removal of eye activity artifacts from visual event-related potentials in normal and clinical subjects. Clinical Neurophysiology, 111(10):1745-1758.

Kinouchi, Y., Ohara, G., Nagashino, H., Soga, T., Shichijo, F., and Matsumoto, K. (1996). Dipole 
source localization of MEG by BP neural networks. Brain Topogr., 8(3):317-321.

Lewine, J. D. and Orrison, II, W. W. (1995). Magnetoencephalography and magnetic source imaging. In Orrison, II, W. W., Lewine, J. D., Sanders, J. A., and Hartshorne, M. F., editors, Functional Brain Imaging, pages 369-417. Mosby, St. Louis.

Makeig, S., Bell, A. J., Jung, T.-P., and Sejnowski, T. J. (1996). Independent component analysis of electroencephalographic data. In Advances in Neural Information Processing Systems 8, pages 145-151. MIT Press.

Makeig, S., Jung, T. P., Bell, A. J., Ghahremani, D., and Sejnowski, T. J. (1997). Blind separation of auditory event-related brain responses into independent components. Proc. Natl. Acad. Sci. USA, 94(20):10979-10984.

Makeig, S., Townsend, J., Jung, T.-P., Enghoff, S., Gibson, C., Sejnwoski, T. J., and Courchesne, E. (1999a). Early visual evoked response peaks apper to be sums of activity in multiple alpha sources. Soc. Neurosci. Abstr., 25(652.8).

Makeig, S., Westerfield, M., Jung, T.-P., Covington, J., Townsend, J., Sejnwoski, T. J., and Courchesne, E. (1999b). Functionally independent components of the late positive event-related potential during visual spatial attention. J. Neurosci., 19(7):2665-2680.

Makeig, S., Westerfield, M., Townsend, J., Jung, T.-P., Courchesne, E., and Sejnowski, T. J. (1999c). Functionally independent components of the early event-related potential in a visual spatial attention task. Philosophical Transactions of the Royal Society: Biological Sciences, 354:1135-44.

Mosher, J. C. and Leahy, R. M. (1998). Recursive music: A framework for EEG and MEG source localization. IEEE Transactions on Biomedical Engineering, 45(11):1342-1354.

Mosher, J. C. and Leahy, R. M. (1999). Source localization using recursively applied projected (RAP) MUSIC. IEEE Transactions on Signal Processing, 47(2):332-340.

Mosher, J. C., Lewis, P. S., and Leahy, R. M. (1992). Multiple dipole modeling and localization from spatiotemporal MEG data. IEEE Transactions on Biomedical Engineering, 39(6):541-557.

Nagano, T., Ohno, Y., Uesugi, N., Ikeda, H., Ishiyama, A., and Kasai, N. (1998). Multi-source localization by genetic algorithm using MEG. IEEE Transactions on Magnetics, 34(5/pt.1):29762979.

Pearlmutter, B. A. and Parra, L. C. (1996). A context-sensitive generalization of ICA. In International Conference on Neural Information Processing, pages 151-157, Hong Kong. SpringerVerlag.

Ribary, U., Ioannides, A. A., Singh, K. D., Hasson, R., Bolton, J. P. R., Lado, F., Mogilner, A., and Llinas, R. (1991). Magnetic-field tomography of coherent thalamocortical 40-Hz oscillations in humans. Proc. Natl. Acad. Sci. USA, 88(24):11037-11041.

Schmidt, D. M., George, J. S., and Wood, C. C. (1999). Bayesian inference applied to the electromagnetic inverse problem. Human Brain Mapping, 7(3):195-212.

Schwartz, D. P., Badier, J. M., Bihoue, P., and Bouliou, A. (1999). Evaluation of a new MEG-EEG spatio-temporal localization approach using a realistic source model. Brain Topogr., 11(4):279- 
289.

Sekihara, K., Nagarajan, S. S., Poeppel, D., Miyauchi, S., Fujimaki, N., Koizumi, H., and Miyashita, Y. (2000). Estimating neural sources from each time-frequency component of magnetoencephalographic data. IEEE Transactions on Biomedical Engineering, 47(5):642-653.

Sekihara, K., Poeppel, D., Marantz, A., Koizumi, H., and Miyashita, Y. (1997). Noise covariance incorporated MEG-MUSIC algorithm: A method for multiple-dipole estimation tolerant of the influence of background brain activity. IEEE Transactions on Biomedical Engineering, 44(9):839-847.

Tang, A. C., Pearlmutter, B. A., Zibulevsky, M., and Carter, S. A. (2000a). Blind separation of multichannel neuromagnetic responses. Neurocomputing, 32-33:1115-1120.

Tang, A. C., Pearlmutter, B. A., Zibulevsky, M., Hely, T. A., and Weisend, M. P. (2000b). An MEG study of response latency and variability in the human visual system during a visual-motor integration task. In Advances in Neural Information Processing Systems 12, pages 185-191. MIT Press.

Tesche, C. D. and Karhu, J. (1997). Somatosensory evoked magnetic fields arising from sources in the human cerebellum. Brain Research, 744(1):23-31.

Uutela, K., Hamalainen, M., and Salmelin, R. (1998). Global optimization in the localization of neuromagnetic sources. IEEE Transactions on Biomedical Engineering, 45(6):716-723.

Vigário, R., Jousmäki, V., Hämäläinen, M., Hari, R., and Oja, E. (1998). Independent component analysis for identification of artifacts in magnetoencephalographic recordings. In Advances in Neural Information Processing Systems 10. MIT Press.

Vigário, R., Särelä, J., Jousmäki, V., Hämäläinen, M., and Oja, E. (2000). Independent component approach to the analysis of EEG and MEG recordings. IEEE Transactions on Biomedical Engineering, 47(5):589-593.

Vigário, R., Särelä, J., Jousmaki, V., and Oja, E. (1999). Independent component analysis in decomposition of auditory and somatosensory evoked fields. In Proc. First International Conference on Independent Component Analysis and Blind Source Separation ICA'99, pages 167-172, Aussois, France.

Wübbeler, G., Ziehe, A., Mackert, B.-M., Müller, K.-R., Trahms, L., and Curio, G. (2000). Independent component analysis of non-invasively recorded cortical magnetic DC-fields in humans. IEEE Transactions on Biomedical Engineering, 47(5):594-599.

Zibulevsky, M. and Pearlmutter, B. A. (2001). Blind source separation by sparse decomposition in a signal dictionary. Neural Computation, 13(4):863-882.

Ziehe, A., Müller, K.-R., Nolte, G., Mackert, B.-M., and Curio, G. (2000). Artifact reduction in magnetoneurography based on time-delayed second order correlations. IEEE Transactions on Biomedical Engineering, 47(1):75-87. 


\section{A Experimental details}

Continuous 122-channel data were collected during the entire period of the following four tasks sampled at $300 \mathrm{~Hz}$ and band-pass filtered at $0.03-100 \mathrm{~Hz}$. A total of four visual reaction time tasks were performed by each subject.

In all tasks, each trial consisted of a pair of colored abstract block compositions, one of which was the target, presented symmetrically and simultaneously on the left and right halves of the screen. Subjects were instructed to respond as quickly and as accurately as possible with a left or right hand mouse button press when the target stimulus was presented to the left or right side of the display screen respectively. The button press elicited an auditory feedback indicating whether a correct or incorrect response was made.

Stimuli were either presented on a 15" VGA computer monitor at a distance of 48 " and occupying $7.6^{\circ}$ of visual angle or back-projected by an LCD projector positioned so that the stimulus occupied the same visual angle. In all tasks the interval between the motor-response and the next stimulus presentation was $3.0 \pm 0.5 \mathrm{~s}$. Auditory feedback was composed of $2000 \mathrm{~Hz}$ and $500 \mathrm{~Hz}$ tones indicating correct and incorrect choices respectively.

The four tasks differed from each other primarily in their definition of the target stimulus which affected how much processing was required for target determination. The precise duration of each task varied slightly across subjects, depending upon the subject's reaction time. Therefore typical durations are given below. The first task (stimulus pre-exposure) consisted of 270 trials. It took the subjects approximately 30 minutes to perform this task. The other three tasks (elemental discrimination, trump card, and transverse patterning) each consisted of 90 trials that were subsets of the same stimuli contained in the first task. Each of the these three tasks took approximately 10 minutes to complete. For each subject, all four experiments were performed on the same day but each in a separate session. Instructions for each experiment were given immediately prior to that experiment. Subjects were permitted to move between experiments. Head positions were recalibrated at the beginning of each experiment. Subjects performed the four experiments in order of increasing task demand: stimulus pre-exposure, trump-card task, elemental discrimination task, and transverse patterning task.

\section{A.1 Stimulus pre-exposure task}

There were no pre-defined relationships between stimuli and button presses. No feedback was given to the subjects about any choice. The subject was instructed to examine both stimuli and then make a roughly equal number of right and left button presses, without consistent alternation between right and left responses. The sequence of presentation was random. Presentations of each stimuli on the left and right sides of the video screen were counterbalanced.

\section{A.2 Trump Card Task}

Subjects were instructed to discover by trial and error which of the two stimuli in the stimulus pair was the target (the trump card). A total of 9 stimulus pairs involving 10 stimuli were used, with a single stimulus as the trump card. Subjects did not have any problem in discovering the trump-card within a few trials. 


\section{A.3 Elemental Discrimination Task}

Subjects were instructed to discover which one of the stimulus pair was the target stimulus by trial and error. A total of three stimulus pairs consisting of six stimuli were used. For each pair of stimuli, one of the pair was the target. This task differs from the trump card task in that multiple target stimuli were involved. All subjects found the targets within a few trials.

\section{A.4 Transverse Patterning Task}

Subjects were instructed to discover which of the two stimuli in a stimulus pair was the target. Three stimulus pairs consisting of three stimulus compositions were used. Each stimulus could be a target or non-target depending upon what it was paired with. The target definition was a "rockpaper-scissors" arrangement: A wins when paired with $\mathrm{B}, \mathrm{B}$ wins when paired with $\mathrm{C}$, $\mathrm{C}$ wins when paired with A. Again, subjects were able to discover the winning relationships after a few trials.

\section{B Mathematical Methods}

\section{B.1 The SOBI Source Separation Algorithm}

The SOBI algorithm proceeds in two stages. First, the sensor signals are zero-meaned and presphered as follows:

$$
\mathbf{y}(t)=\mathbf{B}(\mathbf{x}(t)-\langle\mathbf{x}(t)\rangle)
$$

The angle brackets $\langle\cdot\rangle$ denote an average over time, so the subtraction guarantees that $\mathbf{y}$ will have a mean of zero. The matrix $\mathbf{B}$ is chosen so that the correlation matrix of $\mathbf{y}$, namely $\left\langle\mathbf{y}(t) \mathbf{y}(t)^{T}\right\rangle$, becomes the identity matrix. This is accomplished by moving to the PCA basis using $\mathbf{B}=$ $\operatorname{diag}\left(\lambda_{i}^{-1 / 2}\right) \mathbf{U}^{T}$, where $\lambda_{i}$ are the eigenvalues of the correlation matrix $\left\langle(\mathbf{x}(t)-\langle\mathbf{x}(t)\rangle)(\mathbf{x}(t)-\langle\mathbf{x}(t)\rangle)^{T}\right\rangle$ and $\mathbf{U}$ is the matrix whose columns are the corresponding eigenvectors, i.e. the "PCA components" of $\mathbf{x}$. (This pre-sphering is solely for the purpose of improving the numerics of the situation by constraining the matrix $\mathbf{V}$ below to be a rigid rotation.)

For the second stage, one constructs a set of matrices which, in the correct separated basis, should be diagonal. In our case, we chose a set of time-delay values $\tau$ to compute symmetrized correlation matrices between the signal $\mathbf{y}(t)$ and a temporally shifted version of itself,

$$
\mathbf{R}_{\tau}=\operatorname{sym}\left(\left\langle\mathbf{y}(t) \mathbf{y}(t+\tau)^{T}\right\rangle\right)
$$

where $\operatorname{sym}(\mathbf{M})=\left(\mathbf{M}+\mathbf{M}^{T}\right) / 2$ is a function that takes an asymmetric matrix and returns a closely related symmetric one. This symmetrization discards some information, but the problem is already highly over-constrained, and the symmetrized matrices provide valid, albeit slightly weaker, constraints on the solution.

After calculating the $\mathbf{R}_{\tau}$, we look for a rotation $\mathbf{V}$ that jointly diagonalizes all of them by minimizing $\sum_{\tau} \sum_{i \neq j}\left(\mathbf{V}^{T} \mathbf{R}_{\tau} \mathbf{V}\right)_{i j}^{2}$, the sum of the squares of the off-diagonal entries of the matrix 
products $\mathbf{V}^{T} \mathbf{R}_{\tau} \mathbf{V}$ via an iterative process (Cardoso and Souloumiac, 1996). ${ }^{13}$ The final estimate of the separation matrix is $\mathbf{W}=\mathbf{V}^{T} \mathbf{B}$, which is used to calculate the separated components $\hat{\mathbf{s}}(t)=\mathbf{W} \mathbf{x}(t)$.

\section{B.2 Separated Components in Sensor Space}

Since $\mathbf{W}$ is the estimated unmixing matrix, let us use $\hat{\mathbf{s}}(t)=\mathbf{W} \mathbf{x}(t)$ for the consequent estimated sources, and $\hat{\mathbf{A}}=\mathbf{W}^{-1}$ for the corresponding estimated mixing matrix. Using these, the sensor signals resulting from just one of the components can be computed as $\hat{\mathbf{x}}(t)=\hat{\mathbf{A}} \mathbf{D} \mathbf{W} \mathbf{x}(t)=$ $\hat{\mathbf{A}} \mathbf{D} \hat{\mathbf{s}}(t)$, where $\mathbf{D}$ is a matrix of zeros except for ones on the diagonal entries corresponding to each component which is to be retained.

To localize a single component, one computes

$$
\hat{\mathbf{x}}^{(i)}(t)=\hat{s}_{i}(t) \hat{\mathbf{a}}^{(i)}
$$

where $\hat{\mathbf{a}}^{(i)}$ is the $i^{t h}$ column of $\hat{\mathbf{A}}$ and $\hat{\mathbf{x}}^{(i)}(t)$ is the sensor-space image of source $i$. Because $\hat{\mathbf{x}}^{(i)}(t)$ is at each point in time equal to the unchanging vector $\hat{\mathbf{a}}^{(i)}$, scaled by the time course $\hat{s}_{i}(t)$, dipole fitting algorithms will localize $\hat{\mathbf{x}}^{(i)}(t)$ to the same location no matter what window in time is chosen.

\section{B.3 Scaling}

Blind source separation leaves the freedom to choose an arbitrary scale factor for each component. For instance, the source $s_{i}(t)$ could be scaled up by a factor of ten, and the $i^{\text {th }}$ column of $\mathbf{A}$ scaled down by the same factor of ten, giving rise to the exact same observation $\mathbf{x}(t)$. Making a reasonable assumption that all the sensors have intrinsic Gaussian noise of the same magnitude, we used the additivity of these independent sensor noise to scale each row of $\mathbf{W}$ to give each row a vector length of one. That is, if $\widetilde{\mathbf{W}}$ is the unscaled unmixing matrix, then we normalized its rows to yield W using

$$
w_{i j}=\frac{\tilde{w}_{i j}}{\sqrt{\sum_{j} \tilde{w}_{i j}^{2}}}
$$

With this scale factor, the sources can be viewed as being measured by a "virtual sensor" that measures in the same units, with the same scale, and with the same amount of intrinsic noise as the real sensors.

An alternative approach to scaling is to try to calculate the actual strength of the source, for instance the actual total energy emitted. This can be done by fitting a physical source model (such as an equivalent current dipole) to each component and scaling the rows of $\mathbf{W}$ such that the columns of $\hat{\mathbf{A}}$ match the attenuations predicted by the estimated physical model. This approach has the disadvantage of being dependent on the localization process, thus giving rise to multiple scalings when there are multiple localization procedures in use, or even when a single procedure produces multiple possible localizations. Another disadvantage of this alternative is a failure to generate a scaling when the localization fails, as it would on noise components.

\footnotetext{
${ }^{13}$ Using MATLAB code available at http://sig.enst.fr/ cardoso/.
} 


\section{B.4 Energy / Variance accounted for}

A commonly used statistic is the energy in a source, or the amount of variance it accounts for. The energy of source $i$ is

$$
E_{i}=\sum_{t} \sum_{j}\left(\hat{x}_{j}^{(i)}(t)-\overline{\hat{x}_{j}^{(i)}}\right)^{2}
$$

where the mean is being subtracted to discount DC offsets, an important consideration in MEG. Because the rows of the matrix $\mathbf{W}$ are normalized, we can simplify this expression using Equation 4 yielding

$$
E_{i}=\sum_{t}\left(\hat{s}_{i}(t)-\overline{\hat{s}_{i}}\right)^{2}
$$

which is computationally more efficient. In this paper, we gave the fraction of variance accounted by the $i^{\text {th }}$ component as $E_{i} / \sum_{i} E_{i}$. 